\title{
Modeling of the Quenching of Blast Products from Energetic Materials by Expansion into Vacuum
}

\author{
Sungjin Choi $^{\mathrm{a}}$, D. Scott Stewart ${ }^{\mathrm{a}, *}$, Sunhee Yoo ${ }^{\mathrm{b}}$ \\ ${ }^{a}$ Department of Mechanical Science and Engineering, University of Illinois at Urbana Champaign, IL 60801-2906, USA \\ ${ }^{b}$ Jacobs Engineering, Munitions Directorate, Eglin AFB, FL
}

\begin{abstract}
Condensed phase energetic materials include propellants and explosives. Their detonation or burning products generate dense, high pressure states that are often adjacent to regions that are at vacuum or nearvacuum conditions. An important chemical diagnostic experiment is the time of flight mass spectroscopy experiment that initiates an energetic material sample via an impact from a flyer plate, whose products expand into a vacuum. The rapid expansion quenches the reaction in the products so that the products can be differentiated by molecular weight detection as they stream past a detector. Analysis of this experiment requires a gas dynamic simulation of the products of a reacting multi-component gas that flows into a vacuum region. Extreme computational difficulties can arise if flow near the vacuum interface is not carefully and accurately computed. We modify an algorithm proposed by Munz, [1] that computed the fluxes appropriate to a gas-vacuum interface for an inert ideal gas, and extend it to a multi-component mixture of reacting chemical components reactions with general, non-ideal equations of state. We illustrate how to incorporate that extension in the context of a complete set of algorithms for a general, cell-based flow solver. A key step is to use the local exact solution for an isentropic expansion fan, for the mixture that connects the computed flow states to the vacuum. Regularity conditions (i.e the Liu-Smoller conditions) are necessary conditions that must be imposed on the equation of state of the multicomponent fluid in the limit of a vacuum state. We shows that the Jones, Wilkins, Lee (JWL) equation of state meets these requirements.
\end{abstract}

Keywords: Vacuum Riemann problem; vacuum tracking; multi-component reacting flow; time of flight mass spectroscopy; PETN; JWL; Mie-Gruneisen equation of state

\section{Introduction}

Condensed phase energetic materials include propellants and explosives. They are usually composed of a mixture of granular solids that include explosive or oxidizing crystallites, various metal powders like aluminum, sometimes carbon black, resins and plastics. The performance of the aggregate composite depends on the chemistry and the mechanisms of energy release, which occur in nearly all phases of materials, gas, liquid and solid. Propellant and explosives reactive decomposition produces huge volume expansion. The products start at near solid densities and at high pressures and expand to very low densities and lower pressures. In the case of explosives, the pressure drops from 100's of kilo-bars to 1 atmosphere or less, which is 5 to 6 orders of magnitude across a reaction zone that is often no more than $1 / 10$ of a millimeter thick. This enormous pressure gradient provides the means to cut materials or drive surrounding materials to large velocities by virtue of this expansion power. Likewise, solid propellants in rocket motors vent gases with pressures that range from 1 to 200 atmospheres, down to vacuum conditions. Many orders of magnitudes of pressure change are realized and the pressure gradient in propellant exhaust stream provides the means

${ }^{*}$ Corresponding author: Mechanical and Science Engineering, University of Illinois at Urbana Champaign, IL 60801, USA

Email addresses: dss@illinois.edu (D. Scott Stewart), syoo1@illinois.edu (Sunhee Yoo) 
to generate thrust. The problem of computing the transition of material states from very high pressure to vacuum or near vacuum states is a generic one for any multi-material simulation where two materials may collide and have individual or shared boundaries, for which one of the materials is adjacent to a region with very low pressure and density or a vacuum. Difficulties generally arise if the region of expansion between the high and low pressure regions is not accurately computed.

A time of flight mass spectrometry (TOFMS) experiment by Fossum et al. [2], initiated small samples of energetic materials by laser flyer plate impact. After impact, the products expanded into a vacuum region where spectroscopic analysis of the products was performed. A larger version of a similar experiment was carried out by Blais et al. [3] that used a large quantity of explosive to drive an ampule of nitromethane, which detonated upon being shocked. The premise of the TOFMS experiments is that the pressure drop caused by the expansion in a long vacuum region freezes/quenches the reaction amongst product species in a sequential manner, ordered by the events in the reaction zone in the material that was established prior to the expansion. In order to interpret TOFMS experiments, accurate model simulations of the gas dynamics of reacting multi-component mixtures are required. The range of thermodynamic states is extreme. The reactants start at standard room pressure and temperature conditions, are raised to high pressure, density detonation or highly shocked states in condensed materials, convert to (mostly) gas species products that expand to a vacuum or near-vacuum state. Such simulations are very challenging to carry out since non-ideal equations of state forms must be used for the reactants and products, and the location of the vacuum/materials boundary must be calculated in a precise manner.

Computational methods and techniques are not widely available for multi-component reactive flow, when the components are subjected such huge ranges in the thermodynamics states. Since we had an interest in finding an accurate and robust way to simulate the TOFMS experiments, we decided to make a modification to the basic vacuum interface tracking algorithm pioneered by Munz [1]. Our extension is used in combination with a (now) standard, higher order, cell-based, totally variation diminishing (TVD) Euler scheme, in a fairly general multicomponent framework. One should be able to use our method to compute the approach to the vacuum in the continuum limit, and combine it with simulations of the Boltzmann equation or with molecular dynamic simulations. The continuum simulations we describe would generate the near vacuum continuum flows as a far-field limit, or be used to establish averaged initial conditions for molecular based simulations. In this paper, we describe these algorithms and implementations in detail, and present worked examples that simulate the flow in the TOFMS experiment as a targeted application.

Figure 1 shows a sketch of a typical sequence of events that occur in a simulation of the quenching of blast products that expand into a vacuum region on the right. a) The explosive sample and vacuum regions are initially separated, prior to impact by the flyer. b) If the sample detonates, a detonation shock and its supporting reaction zone propagate through the explosive. c) The detonation wave hits the vacuum interface. d) Then a rarefaction travels back into the detonation reaction zone structure. As the pressure and density drop, the reactions amongst product species slow as the products flow into the vacuum section, past the detector which monitors the mass concentration of the products at a fixed probe locations. Since the number density drops in the vacuum region, the species are thought not to undergo significant collisions and hence no further chemical changes occur.

\subsection{Summary of the algorithms}

The original work of Munz [1] proposed an approximation method that computes the fluxes, appropriate to the gas-vacuum interface, and tracked the motion of the gas-vacuum boundary for an inert ideal gas. Our extended algorithm allows for reactive flow with general, non-ideal equation of state (EOS) in Mie-Gruneisen (MG) form for a reacting multi-component mixture, adjacent to a vacuum interface. The formulation can be used to include an arbitrary number of chemical components or other state variables like porosity or particulate density, that could be included in the constitutive description. Our standard multi-component flow model has only one material velocity for the mixture. The corresponding gas dynamics formulations have only forward, backward acoustic characteristic and one material characteristic. The exact form of the vacuum Riemann problem can thus be handled in a standard and familiar way, albeit with complexities associated with the EOS that we describe in some detail. We note that Lee, et. al. [4] recently considered the Riemann problem for the MG EOS in their analysis of difficulties associated with contact discontinuities. 


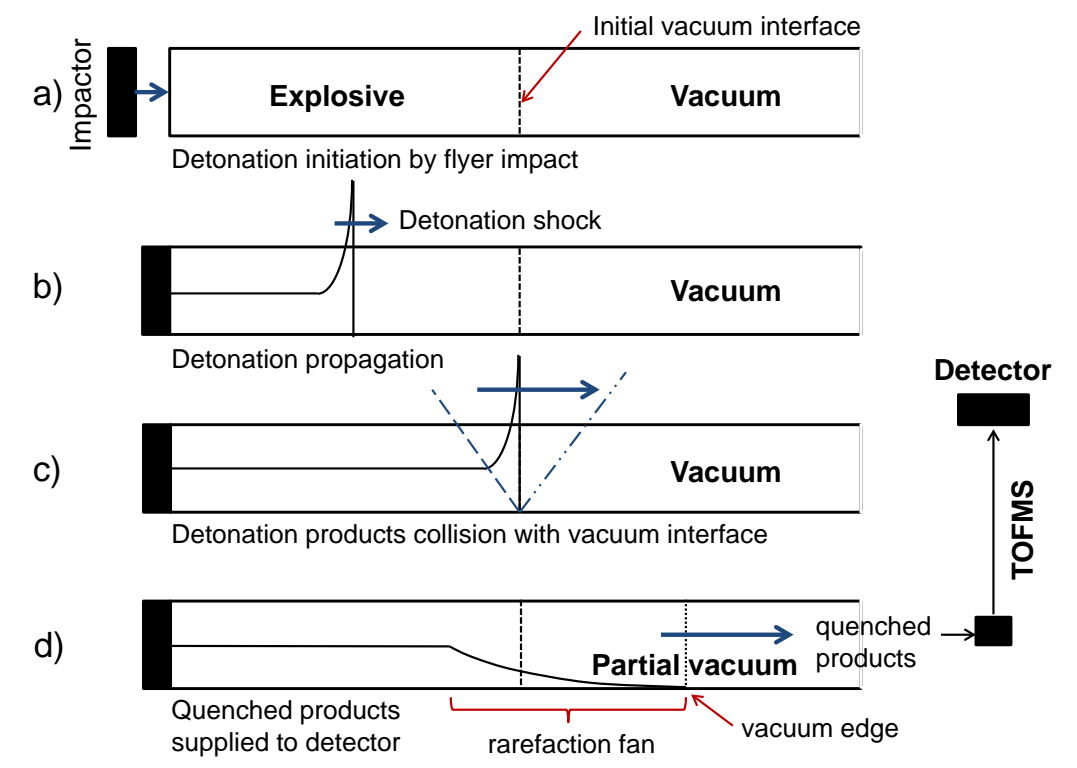

Figure 1: The schematic diagram of numerical simulation of initiation, detonation and expansion for an explosive sample in the TOFMS experiment.

The extended vacuum tracking algorithm is described in the context of other supporting methods and solver algorithms. We use a cell-based reactive Euler solver that employs local cell-based Riemann problems for general EOS to compute the cell edge fluxes. Roe-average fluxes are used in the manner originally developed by Glaister, [5] and extended by $\mathrm{Xu}$ [6], and $\mathrm{Xu}$, Aslam and Stewart [7] for chemically reacting species. The reactive Euler equations are solved by source splitting with the first update as a solution to the Euler equations without source term, followed by an update of the (chemical) source terms. We will reference to the first update as the hydrodynamic update and the second update as the source term update. We have used $5^{t h}$ order Weighted Essentially Non Oscillatory (WENO) with 3rd order Runge Kutta (RK) for the hydrodynamic update. Updates to the location and velocity of vacuum interface of material, and the special cell boundary flux calculations, use the extensions to the Munz [1] tracking scheme. We have verified our schemes against various examples found in the literature $[1,5,8]$.

Care must be taken to accurately integrate the chemical source terms, because the rates in most kinetics schemes widely vary and can have both fast and slow time scales that are disparate. Colella et al. [9] were amongst the first to point this out for Arrhenius kinetics, applied to the calculation of gas-phase reacting flow. In this case it was advantageous to analytically integrate an approximate form of the source terms across a time step. However source term splitting treatments have been proven to work well (dating back to Colella's original work) and are known to have good stability properties. In his thesis Xu [6] demonstrated that an algorithm of the same type we use here for non-ideal EOS forms, was second order accurate.

If one uses an inaccurate numerical method for the basic hydrodynamic solver, significant problems arise from inaccuracies in the evaluation of the sound speed $c=\sqrt{\partial p /\left.\partial \rho\right|_{s}}$ in the vicinity of the vacuum region, in which case tracking the vacuum material interface becomes difficult or breaks down. Therefore in order to compute the flux state and velocity of the vacuum material interface, we work out the exact form of the isentropic expansion fan that vents to the interface. This connects the vacuum state to the state in the adjacent cell, and thus supplies the combined method with a physically accurate value for the vacuum interface velocity. Since we allow for general equation of state, we must insist that the limiting behaviors of the EOS forms are such that the vacuum/material interface problem is well-posed. Fortunately, the conditions that define a well-posed vacuum Riemann problem (VRP) have been considered by Liu and Smoller [10] and they provide criteria on limiting functional form of the EOS as the material expands to 
the vacuum state of zero mass density. These conditions must be incorporated into the vacuum interface tracking method. We show that the Jones, Lee, Wilkins (JWL) EOS form, commonly used for product components, satisfies these conditions.

\subsection{Paper outline}

Section 2 describes basic model formulations that include the conservation laws, the mixture equation of state for a multi-component mixture with commonly used EOS forms for the components, that include ideal gas EOS forms and Mie-Gruneisen EOS forms. Details are given for the JWL EOS form for reactant and product components. Details for two component mixture closures are given. One is the standard pressuretemperature equilibrium closure (PT-EQB), and the other is an often-used, simpler, pressure equilibrium closure (P-EQB), that specifies a volume ratio between the components( instead of enforcing strict temperature equilibrium). Section 3 describes the numerical methods, with a brief review of basic methods for the compressible Euler equations. A brief description of the Riemann solver for general EOS is given followed by the detailed discussion of the implementation of the vacuum tracking method for a general EOS in a reactive flow. Section 4 presents examples and applications. The first example describes a condensed explosive with ideal gas EOS forms for both the reactants and products. This is followed by a detailed simulation of a facsimile of the TOFMS experiment where a thin sample of PETN is hit by an impactor and whose products are vented into the long vacuum region of the experiment. The components are modeled with non-ideal MG EOS forms. We also discuss the sensitivity of the simulation results to the different mixture closure conditions, pressure, temperature equilibrium (PT-EQB) and pressure equilibrium (P-EQB).

\section{Basic model formulations}

Here we give the formulations for the physical models of interest. The models apply to a mixture of compressible fluids with $N$ distinct species. The fluid mixture is assumed to have a single mixture material velocity $u$, pressure $p$, and generally a single temperature $T$, (although that is not strictly required). Each species will be assumed to be specified by its separate equation of state, and the mixture energy that is used to define the equilibrium potentials will neglect mixing terms, that can sometime be included in the theory of liquid mixtures. Hence the mixture is ideal, although the equation of state (EOS) forms are not necessarily those of the ideal gases. We assume that the EOS forms provided by the model allow for a continuous and smooth approach to zero density, i.e to the vacuum state. That will allow us to calculate reasonable mixture velocities and track the location of vacuum material interface. It is understood that the continuum limit breaks down at a true vacuum and the model only predicts some limiting approximation (from the continuum limit side) of the states near the material/vacuum interface. The description of the transition to vacuum on the molecular scale requires the implementation of additional models, that are not the subject of this work. Thus the flow simulations as shown in the section for examples are restricted to extremely low but non-zero densities, where the continuum approximations are still reasonable.

\subsection{The governing equations}

It is useful to present all three forms of the governing equations, the primitive, conservation and characteristic forms. The basic numerical scheme in the body of the fluid uses the conservative form, while the vacuum interface approximation require the characteristic form. The primitive form of the governing conservation laws for mixture mass, momentum, energy, and mass fraction of identified chemical species are respectively

$$
\begin{gathered}
\dot{\rho}+\rho \nabla \cdot \boldsymbol{v}=0, \\
\rho \dot{\boldsymbol{v}}=-\nabla p, \\
\rho \dot{e}+p \nabla \cdot \boldsymbol{v}=0, \\
\rho \dot{\lambda_{i}}=r_{\lambda_{i}},
\end{gathered}
$$


where we use the "dot" notation is used for the material derivative $(\dot{)}=\partial / \partial t+\boldsymbol{v} \cdot \nabla$. The variables $\rho=1 / v, e, \boldsymbol{v}$ and $\lambda_{i}$ are the mixture density (its reciprocal, specific volume), specific internal energy, mixture velocity and mass fraction of the ith-species respectively. The one-dimensional conservative form of the Euler equations for reactive flow in the multi-component mixture (illustrated for two independent components/reactions, $\left.\lambda_{1}, \lambda_{2}\right)$ are:

$$
\begin{gathered}
\frac{\partial \mathbf{U}}{\partial t}+\frac{\partial \mathbf{F}(\boldsymbol{U})}{\partial x}=\mathbf{S}(\mathbf{U}) \\
\mathbf{U}=\left[\begin{array}{c}
\rho \\
\rho u \\
\rho E \\
\rho \lambda_{1} \\
\rho \lambda_{2}
\end{array}\right] \quad \mathbf{F}=\left[\begin{array}{c}
\rho u \\
\rho u^{2}+p \\
(\rho E+p) u \\
\rho u \lambda_{1} \\
\rho u \lambda_{2}
\end{array}\right] \quad \boldsymbol{S}=\left[\begin{array}{c}
0 \\
0 \\
0 \\
\rho r_{\lambda_{1}} \\
\rho r_{\lambda_{2}}
\end{array}\right],
\end{gathered}
$$

and

$$
E=e+\frac{1}{2} u^{2}
$$

Riemann problems and the methods for solving these hyperbolic equations are based on the characteristic form of the equations which we list below in one spatial dimension, $x$

$$
\begin{gathered}
\frac{\partial p}{\partial t}+(u+c) \frac{\partial p}{\partial x}+\rho c\left[\frac{\partial u}{\partial t}+(u+c) \frac{\partial u}{\partial x}\right]=\rho c^{2}(\boldsymbol{\sigma} \cdot \boldsymbol{r}), \\
\frac{\partial p}{\partial t}+(u-c) \frac{\partial p}{\partial x}-\rho c\left[\frac{\partial u}{\partial t}+(u-c) \frac{\partial u}{\partial x}\right]=\rho c^{2}(\boldsymbol{\sigma} \cdot \boldsymbol{r}), \\
\frac{\partial p}{\partial t}+u \frac{\partial p}{\partial x}-c^{2}\left(\frac{\partial \rho}{\partial t}+u \frac{\partial \rho}{\partial x}\right)=\rho c^{2}(\boldsymbol{\sigma} \cdot \boldsymbol{r}), \\
\frac{\partial \lambda_{i}}{\partial t}+u \frac{\partial \lambda_{i}}{\partial x}=r_{\lambda_{i}} .
\end{gathered}
$$

The sound speed $c^{2}$ and the thermicity $\boldsymbol{\sigma} \cdot \boldsymbol{r}$ are given by

$$
c^{2}=\frac{\left(\left.\frac{\partial e}{\partial v}\right|_{p, \lambda_{i}}+p\right) v^{2}}{\left(\left.\frac{\partial e}{\partial p}\right|_{v, \lambda_{i}}\right)} \quad \text { with } \quad \sigma_{i}=\frac{1}{\rho c^{2}} \frac{\left.\left(\partial e / \partial \lambda_{i}\right)\right|_{p, v}}{\left.(\partial e / \partial p)\right|_{v, \lambda_{i}}} \text { and } \boldsymbol{\sigma} \cdot \boldsymbol{r}=\sum_{i}^{N} \sigma_{i} r_{\lambda_{i}},
$$

respectively, and where the specific internal energy is of the form $e\left(p, v, \lambda_{i}\right)$. The equations above, written as ordinary differential equations on their respective characteristics, are given by

$$
\begin{aligned}
& \frac{d p}{d t}+\rho c \frac{d u}{d t}=\rho c^{2}(\boldsymbol{\sigma} \cdot \boldsymbol{r}) \quad \text { on } C_{+}: \quad \frac{d x}{d t}=u+c, \\
& \frac{d p}{d t}-\rho c \frac{d u}{d t}=\rho c^{2}(\boldsymbol{\sigma} \cdot \boldsymbol{r}) \quad \text { on } C_{-}: \quad \frac{d x}{d t}=u-c, \\
& \frac{d p}{d t}-c^{2} \frac{d \rho}{d t}=\rho c^{2}(\boldsymbol{\sigma} \cdot \boldsymbol{r}) \quad \text { on } C_{0}: \quad \frac{d x}{d t}=u, \\
& \frac{d \lambda_{i}}{d t}=r_{\lambda_{i}} \quad \text { on } C_{0}: \quad \frac{d x}{d t}=u .
\end{aligned}
$$

\subsection{Mixture Equation of State}

We assume that the fluid mixture is comprised of $N$ chemical components. A complete equation of state is supposed to be given for each component (species). One can suppose that a Gibbs free energy form is provided for each component that specifies that energy at a fixed temperature and pressure in the form 
$g_{i}(p, T)$, which is a complete equation of state, Callen [11], from which we can write the specific internal energy and pressure, volume temperature equation of states, in the form

$$
e_{i}\left(p, v_{i}\right) \text { and } v_{i}=v_{i}(p, T) .
$$

The internal energy and volume are extensive thermodynamic quantities, so the contribution from each component add in a fixed volume of mixture in proportion to the relative masses of the components. Additional contributions due to the intermolecular mixing are neglected. If the mass fraction of each component is $\lambda_{i}$, then the specific energies and the volumes of the mixture can be written as the sums

$$
e\left(p, T ; \lambda_{i}\right)=\sum_{i=1}^{N} e_{i}\left(p, v_{i}(p, T)\right) \lambda_{i} \quad \text { and } \quad v\left(p, T ; \lambda_{i}\right)=\sum_{i=1}^{N} v_{i}(p, T) \lambda_{i},
$$

with $\sum_{i=1}^{N} \lambda_{i}=1$. For fixed mixture energy energy and volume and internal composition, equations (18a,b) are implicit equations for the pressure and temperature, $(p, T)$.

We require the regularity conditions on the equation of state, called the Liu-Smoller conditions (see [10]), when the density approaches to zero density on an isentrope. The pressure relations modeled by the mixture EOS (18) should satisfy

$$
p(0)=0,\left.\quad \frac{\partial p(0)}{\partial \rho}\right|_{s}=0, \quad \text { and }\left.\quad \frac{\partial p}{\partial \rho}\right|_{s}>0,\left.\quad \frac{\partial^{2} p}{\partial \rho^{2}}\right|_{s}>0 \quad \text { for } \quad \rho>0 .
$$

In order to model a particular energetic or explosive material, one must identify all the components and their EOS forms for each component. The ideal gas EOS form is often used in detonation theory, [12], and is also the EOS form that was used by Munz to establish his original vacuum tracking algorithm. We describe those common EOS forms next.

\subsubsection{Ideal gas EOS forms for the components}

When all the components are specified by the ideal EOS form with constant specific heats of formation, we write

$$
e_{i}=\frac{1}{\gamma_{i}-1} p v_{i}+e_{i 0}
$$

where $\gamma_{i}=c_{p i} / c_{v i}$ (the ratio of specific heats) and $e_{i 0}$ is the heat of formation of the $\mathrm{i}$-th component. We also use the notation $\omega_{i} \equiv\left(\gamma_{i}-1\right)$. The component gas constant is $R_{i}=c_{v i}\left(\gamma_{i}-1\right)$, and the $p, v_{i}, T$ EOS is given by

$$
p v_{i}=R_{i} T
$$

The mixture energy and mixture volume are given by

$$
e=p \sum_{i} \frac{v_{i}}{\gamma_{i}-1} \lambda_{i}+\sum_{i} e_{i 0} \lambda_{i}, \quad \text { and } \quad v=\frac{T}{p} \sum_{i} R_{i} \lambda_{i}
$$

Equation (22a) can also be written as

$$
e=\left(\sum_{i} c_{v i} \lambda_{i}\right) T+\sum_{i} e_{i 0} \lambda_{i}
$$

\subsubsection{A Mie-Gruneisen EOS form for components}

A $e(p, v)$ form of Mie-Gruneisen (MG) EOS for components is explicitly linear in the pressure $p$ and generally nonlinear in the specific volume, and can be written (dropping the i-subscript temporarily) as 


$$
e(p, v)=e^{s}(v)+\frac{v}{\Gamma}\left(p-p^{s}(v)\right) \equiv A(v)+G(v) p
$$

The functions $e^{s}(v), p^{s}(v)$, and $v / \Gamma$ are functions that are determined from EOS calibration experiments. Often $e^{s}(v)$ and $p^{s}(v)$ are identified as the internal energy and pressure at reference states on some specified, well-identified and measurable thermodynamic process, such as the states on a $p, v$ expansion isentrope, or a shock Hugoniot. Here they are simply regarded as known functions of the specific volume, $v$. The Mie-Gruneisen coefficient $\Gamma$ is often assumed to be a constant or simply a function of $v$. We note that the ideal EOS forms are in this MG class, so the MG forms are inclusive of the ideal EOS forms.

\subsubsection{The Jones, Lee, Wilkins (JWL) EOS form for components}

The Jones, Lee, Wilkins (JWL) EOS form for components is in the MG class, and is probably the most commonly used EOS form for engineering studies of reactive flow in explosives. For example, the chemical equilibrium code Cheetah [13] automatically generates JWL equation of state for reaction products. The JWL EOS forms are often paired with the "Ignition and Growth" reaction rate models that were pioneered by Lee and Tarver, [14]. Many highly used explosive compounds such as HMX, RDX, PETN, TATB, TNT, etc. are modeled with MG EOS forms, paired with Ignition \& Growth kinetic rate models that describe the rate of overall decomposition from reactants to products.

A standard convention is to use a lower case letter for specific internal energy, (as in $e$ ) and a upper case letter for energy per unit volume $E$. An upper case $V$ to represent the specific volume ratio relative to a reference volume (as in $V=v / v_{0}$ ). Then for each component the JWL and thermal EOS is given by

$$
p=A_{i} \exp ^{-R_{1 i} V_{i}}+B_{i} \exp ^{-R 2 i V_{i}}+\frac{\omega_{i} C_{v i}}{V_{i}} T \quad \text { with } \quad V_{i}=\frac{v_{i}}{v_{0}},
$$

and the JWL EOS form are given by the $e\left(p, v_{i}\right)$ mechanical EOS given by

$$
E_{i}=E_{i}^{s}\left(V_{i}\right)+\frac{V_{i}}{\omega_{i}}\left[p-p_{i}^{s}\left(V_{i}\right)\right]+E_{0 i},
$$

with

$$
E_{i}^{s}(v)=\left(\frac{A e^{-R_{1 i} V_{i}}}{R_{1 i}}+\frac{B e^{-R_{2 i} V_{i}}}{R_{2 i}}\right) \quad \text { and } \quad p_{i}^{s}\left(V_{i}\right)=\left(A_{i} e^{-R_{1 i} V_{i}}+B_{i} e^{-R_{i 2} V_{i}}\right) .
$$

With $e_{i}=E_{i} v_{0}$ one has

$$
e_{i}=e_{i}^{s}\left(v_{i}\right)+\frac{v_{i}}{\omega_{i}}\left[p-p_{i}^{s}\left(v_{i}\right)\right]+e_{0 i}
$$

with $e_{i}^{s}\left(v_{i}\right)=E_{i}^{s} v_{0}$. The constants $A_{i}, B_{i}, R_{1 i}, R_{2 i}, \omega_{i}$ and $C_{v i} \equiv c_{v i} / v_{0}$ are the JWL component EOS constants; $e_{0 i}$ is the reference energy and $v_{0}$ is a standard reference volume of the original, un-shocked mixture.

\subsubsection{A mixture EOS of reactant and product components for the JWL EOS forms}

Next suppose we specify that the mixture is composed of only two species/components reactants (R) and products $(\mathrm{P})$. Both are assumed to be modeled by the JWL EOS forms. The material decomposes by the reaction $R \rightarrow P$, during which the reactant and product are assumed to be in pressure and temperature equilibrium. The product mass fraction is taken be $\lambda$ while the reactant mass fraction be $1-\lambda$. An $R$ subscript is used to denote reactant and $P$ for product. Given the energy $e$ and a mixture volume $v$, the constraints (18) become

$$
\begin{gathered}
e=\lambda e_{P}\left(p, v_{P}(p, T)\right)+(1-\lambda) e_{R}\left(p, v_{R}(p, T)\right) \\
v=\lambda v_{P}(p, T)+(1-\lambda) v_{R}(p, T)
\end{gathered}
$$


Since the JWL forms (25) and (26) are not generally linear in the specific volume, the volume dependence is implicit in $p, T$. Given specified values for $e, v, \lambda$, equations (30) and (29) are two equations for $p$ and $T$. The solution completes the EOS description for the mixture, and defines the PT-EQB closure. There are many ways to find the roots but all involve a iteration to find roots of a pairs (or n-tuples) of equations. For the specific choice of the MG, JWL EOS forms for two components, the problem of finding the $(p, T)$ root pairs can be reduced to a single scalar unknown. Next we present a simple robust algorithm that can be used to find the roots.

\subsubsection{Determination of the $(p, T)$ (Gibbs) equilibrium states}

We present a simple way to compute the PT-EQB for JWL EOS forms, that can be used for $N$ components; illustrated for three that can be extended simply to $n$. The mechanical and thermal EOS forms can be represented as

$$
e_{i}\left(p, v_{i}\right) \text { and } p=p\left(v_{i}, T\right)
$$

for some fixed temperature and pressure, where $e_{i}, v_{i}$ are the specific energy and volume for that component. The mixture energy and volume are represented as a sum over the components

$$
e=\lambda_{1} e_{1}+\lambda_{2} e_{2}+\lambda_{3} e_{3}, \quad \text { and } \quad v=\lambda_{1} v_{1}+\lambda_{2} v_{2}+\lambda_{3} v_{3} .
$$

Use $v_{1}$ as a reference volume and define the component volume ratios

$$
\Phi_{2}=v_{2} / v_{1} \text { and } \Phi_{3}=v_{3} / v_{1}
$$

which combined with $(31 \mathrm{~b})$ solves for the component volumes in term of the mixture volume and the ratios, to obtain

$$
v_{1}=\frac{v}{\lambda_{1}+\lambda_{2} \Phi_{2}+\lambda_{3} \Phi_{3}}, v_{2}=\frac{v \Phi_{2}}{\lambda_{1}+\lambda_{2} \Phi_{2}+\lambda_{3} \Phi_{3}}, \quad v_{3}=\frac{v \Phi_{3}}{\lambda_{1}+\lambda_{2} \Phi_{2}+\lambda_{3} \Phi_{3}} .
$$

Using the forms (28) for $e_{i}$ in (31a) obtains

$$
e=p \sum_{i} \frac{v_{i}}{\omega_{i}} \lambda_{i}+\sum_{i}\left[e_{i}^{s}\left(v_{i}\right)-\frac{v_{i}}{\omega_{i}} p_{i}^{s}\left(v_{i}\right)\right] \lambda_{i}+\sum_{i} e_{i 0} \lambda_{i}
$$

Solving for $p$ gives

$$
p=\left[e-\sum_{i}\left[e_{i}^{s}\left(v_{i}\right)-\frac{v_{i}}{\omega_{i}} p_{i}^{s}\left(v_{i}\right)\right] \lambda_{i}-\sum_{i} e_{i 0} \lambda_{i}\right] /\left[\sum_{i} \frac{v_{i}}{\omega_{i}} \lambda_{i}\right] .
$$

The pressure is now explicitly determined by direct evaluation once the mixture energy $e$ and the volume ratios $\Phi_{2}, \Phi_{3}$ are known. Thus one needs two (2) residuals to determine the correct values of $\Phi_{2}$ and $\Phi_{3}$. Those residuals come from setting the temperature of the component (species) equal. For the case of three components there are two such equivalences, i.e. $T \equiv T_{1}=T_{2}$, and $T_{2}=T_{3}$. For $\mathrm{N}$ components there are $N-1$ volume ratios and, likewise $N-1$ temperature equivalences. For the 3 - component case, with the JWL, MG forms, these equivalences are

$$
\begin{aligned}
& T=\frac{1}{C_{v}^{1}} \frac{V_{1}}{\omega_{1}}\left[p-\left(A_{1} e^{-R_{1}^{1} V_{1}}+B_{1} e^{-R_{2}^{1} V_{1}}\right)\right]=\frac{1}{C_{v}^{2}} \frac{V_{2}}{\omega_{2}}\left[p-\left(A_{2} e^{-R_{1}^{2} V_{2}}+B_{2} e^{-R_{2}^{2} V_{2}}\right)\right], \\
& T=\frac{1}{C_{v}^{3}} \frac{V_{3}}{\omega_{1}}\left[p-\left(A_{3} e^{-R_{1}^{3} V_{3}}+B_{3} e^{-R_{2}^{3} V_{3}}\right)\right]=\frac{1}{C_{v}^{2}} \frac{V_{2}}{\omega_{2}}\left[p-\left(A_{2} e^{-R_{1}^{2} V_{2}}+B_{2} e^{-R_{2}^{2} V_{2}}\right)\right],
\end{aligned}
$$

recast as the pair

$$
\frac{1}{C_{v}^{1}} \frac{V_{1}}{\omega_{1}}\left[p-p_{1}^{s}\left(V_{1}\right)\right]=\frac{1}{C_{v}^{2}} \frac{V_{2}}{\omega_{2}}\left[p-p_{2}^{s}\left(V_{2}\right)\right], \quad \frac{1}{C_{v}^{3}} \frac{V_{3}}{\omega_{3}}\left[p-p_{3}^{s}\left(V_{3}\right)\right]=\frac{1}{C_{v}^{2}} \frac{V_{2}}{\omega_{2}}\left[p-p_{2}^{s}\left(V_{2}\right)\right],
$$


that defines the two residuals. For a two component case only one residual is required.

The roots are determined by the following steps. 1) Specify the mixture values of $v$ and $e$ and the reaction progress variables, $\lambda_{i}$. 2) Make an initial guess for $\Phi_{2}$ and $\Phi_{3}$ (say) and then the values of the component volumes (for the example: $v_{1}, v_{2}$ and $v_{3}$ are computed. 3) Then the residuals defined by (38a, 38b) can be evaluated. 4) Iterate using the residuals to find the root. Any reasonable root procedure can be used. Newton Raphson works well and converges in a few steps. In special cases direct searches can be used. The initial guess for the volume ratios are usually determined from the previous (time step) physical state, so good quality initial guesses are in abundance. The convergence of the root finding procedure then determines all the component ratios, which in turn can be used to directly compute the pressure from (35) and temperature from (36) or (37).

\section{Numerical Methods}

\subsection{The Euler Solver}

A typical numerical solution of our equations would use operator splitting that updates a discrete approximation to the source free Euler equations, followed by an update of the source terms. The latter generally requires a stiff ODE solver that can handle the stiff reaction terms. In our implementation we used the splitting methods described by Toro, [8]. A Roe-type solver is used to update the source free Euler equations, and a stiff ODE package, Livermore Solver of Ordinary Differential Equations (LSODE) [15], is used to update the source terms for interior points in the domain. The first update takes the initial data at the current time $t^{n}$ and solves the source free Euler equations and returns the updated states at $t^{n+1}$. Thus

CYCLE 1: The Hydrodynamic update: Solve

$$
\mathbf{U}_{t}+\mathbf{F}(\mathbf{U})_{x}=0,
$$

subject to the initial condition $\mathbf{U}\left(x, t^{n}\right)=\mathbf{U}^{n}$, and return the solution at $t^{n+1}$ so that $\mathbf{U}^{\text {hydro }} \equiv$ $\mathbf{U}\left(x, t^{n+1}\right)$.

The second source term update uses the first update as the initial conditions at $t^{n}$, and then solves just the temporal ODES with the source terms, to generated the final update at $t^{n+1}$. Thus

CYCLE 2: Source term update: Solve

$$
\frac{d \mathbf{U}}{d t}=\mathbf{S}(\mathbf{U})
$$

subject to the initial condition $\mathbf{U}\left(x, t^{n}\right)=\mathbf{U}^{\text {hydro }}$, and return the solution at $t^{n+1}, \mathbf{U}\left(x, t^{n+1}\right)=$ $\mathbf{U}^{n+1}$.

The hydrodynamics updates $\mathbf{U}^{\text {hydro }}$ obey (39) and their cell state averages $\overline{\mathbf{U}}_{i+1 / 2}^{n}$, centered at $i+1 / 2$, are advanced in the standard way as

$$
\overline{\mathbf{U}}_{i+1 / 2}^{n+1}=\overline{\mathbf{U}}_{i+1 / 2}^{n}-\frac{\Delta t}{\Delta x}\left[\mathbf{F}_{i+1}-\mathbf{F}_{i}\right],
$$

where $\mathbf{F}_{i+1}$ and $\mathbf{F}_{i}$ are numerical fluxes at the cell boundaries. The numerical flux is computed by using Roe's approximation as follows:

$$
\mathbf{F}_{i+1}=\frac{1}{2}\left(\mathbf{F}\left(\mathbf{U}_{L}\right)+\mathbf{F}\left(\mathbf{U}_{R}\right)\right)-\frac{1}{2} \sum_{i}\left|\tilde{\boldsymbol{\Lambda}}_{i}\right| \tilde{\boldsymbol{\alpha}}_{i} \tilde{\boldsymbol{R}}_{i} .
$$

The vectors $\mathbf{U}_{L}$ and $\mathbf{U}_{R}$ in equation (42) are the state vectors at cell interface at $i^{\text {th }}$ grid point and these two vectors were obtained by using WENO scheme, which were first introduced by Harten et al. (see [16]). 
The details of WENO reconstruction are found in $[7,17,18]$ for example. The other quantities $\tilde{\alpha}_{i}, \tilde{\Lambda}_{i}, \tilde{R}_{i}$ in equation (42) are the vector $\boldsymbol{\alpha}$, matrices functions $\boldsymbol{\Lambda}$, and $\boldsymbol{R}$ applied at Roe averaged state $\tilde{\mathbf{U}}$. Those computations require the Jacobian of the exact system given by

$$
\boldsymbol{J}(\mathbf{U})=\frac{\partial \mathbf{F}}{\partial \boldsymbol{U}}=\left[\begin{array}{ccccc}
0 & 1 & 0 & 0 & 0 \\
c^{2}-u^{2}-\frac{p_{, e}}{\rho}\left(H-u^{2}\right)-\frac{\lambda_{1} p_{, \lambda_{1}}}{\rho}-\frac{\lambda_{2} p_{\lambda_{2}}}{\rho} & 2 u-\frac{u p_{, e}}{\rho} & \frac{p, e}{\rho} & \frac{p, \lambda_{1}}{\rho} & \frac{p, \lambda_{2}}{\rho} \\
u\left(c^{2}-H-\frac{p, e}{\rho}\left(H-u^{2}\right)-\frac{\lambda_{1} p_{, \lambda_{1}}}{\rho}-\frac{\lambda_{2} p_{, \lambda_{2}}}{\rho}\right) & H-\frac{u^{2} p_{, e}}{\rho} & u\left(1+\frac{p_{, e}}{\rho}\right) & u \frac{p_{, \lambda_{2}}}{\rho} & u \frac{p_{, \lambda_{2}}}{\rho} \\
-u \lambda_{1} & \lambda_{1} & 0 & u & 0 \\
-u \lambda_{2} & \lambda_{2} & 0 & 0 & u
\end{array}\right],
$$

where

$$
H=e+\frac{1}{2} u^{2}+\frac{p}{\rho} \quad \text { and } \quad c^{2}=p_{, \rho}+\frac{p}{\rho^{2}} p_{, e},
$$

and the comma notation subscript denotes the partial derivative with respect to thermodynamics argument.

The Jacobian can be diagonalized and has real eigenvalues (the characteristic wave speeds) and real eigenvectors according to

$$
\boldsymbol{J}=\boldsymbol{R} \boldsymbol{\Lambda} \boldsymbol{R}^{-1},
$$

where $\boldsymbol{R}$ is identified as the matrix composed the right eigenvectors of $\mathbf{J}$ (columns)

$$
\boldsymbol{R}(\mathbf{U})=\left[\begin{array}{ccccc}
1 & 1 & 1 & 0 & 0 \\
u-c & u & u+c & 0 & 0 \\
H-u c & H-p / \rho-\rho \frac{p_{\rho}}{p_{, e}} & H+u c & -\frac{p, \lambda_{1}}{p, e} & -\frac{p, \lambda_{2}}{p, e} \\
\lambda_{1} & \lambda_{1} & \lambda_{1} & 1 & 0 \\
\lambda_{2} & \lambda_{2} & \lambda_{2} & 0 & 1
\end{array}\right]
$$

and $\boldsymbol{\Lambda}$ is the diagonal matrix corresponding to the eigenvectors

$$
\boldsymbol{\Lambda}=\operatorname{diag}[(u-c), u,(u+c), u, u] .
$$

The Roe averaged states are defined by

$$
\tilde{\mathbf{U}}=\left[\tilde{\rho}, \tilde{u}, \tilde{e}, \tilde{\lambda}_{1}, \tilde{\lambda}_{2}\right]
$$

where

$$
\begin{aligned}
\tilde{\rho} & =\sqrt{\rho_{R} \rho_{L}}, \\
\tilde{u} & =\frac{\sqrt{\rho_{L}} u_{L}+\sqrt{\rho_{R}} u_{R}}{\sqrt{\rho_{L}}+\sqrt{\overline{\rho_{R}}}}, \\
\tilde{e} & =\frac{\sqrt{\rho_{L}} e_{L}+\sqrt{\rho_{R}} e_{R}}{\sqrt{\rho_{L}}+\sqrt{\rho_{R}}}, \\
\tilde{\lambda_{1}} & =\frac{\sqrt{\rho_{L}} \lambda_{1 L}+\sqrt{\rho_{R}} \lambda_{1 R}}{\sqrt{\rho_{L}}+\sqrt{\rho_{R}}}, \\
\tilde{\lambda_{2}} & =\frac{\sqrt{\rho_{L}} \lambda_{2 L}+\sqrt{\rho_{R}} \lambda_{2 R}}{\sqrt{\rho_{L}}+\sqrt{\rho_{R}}} .
\end{aligned}
$$


The enthalpy $H$ in $\boldsymbol{J}(\mathbf{U})$ is also computed in the same way,

$$
\tilde{H}=\frac{\sqrt{\rho_{L}} H_{L}+\sqrt{\rho_{R}} H_{R}}{\sqrt{\rho_{L}}+\sqrt{\rho_{R}}} .
$$

Detailed derivations of Roe average and the pressure derivatives of the averages can be found in Roe, [19, 20], Glaister [5]), $\mathrm{Xu}[6]$ and elsewhere.

\subsection{Vacuum Riemann Problem(VRP) and Vacuum Tracking Method for non-ideal EOS}

The vacuum Riemann problem (VRP) is by definition a Riemann problem with vacuum initial condition on one side and a constant state at another side of a computational domain. The vacuum is defined such that the mass density in the geometrical domain of the vacuum is zero, i.e there is an absence of material. Consider the situation with the presence of vacuum in the right state $(x>0)$. In the vacuum region, the conserved variables are zero so that the sound velocity and pressure will also be zero. The VRP for the source free Euler equations can be written as

$$
\frac{\partial \mathbf{U}}{\partial t}+\frac{\partial \mathbf{F}(\mathbf{U})}{\partial x}=\mathbf{0}
$$

and the initial condition is

$$
\mathbf{U}(x, 0)=\left\{\begin{aligned}
\left(\rho_{L}, u_{L}, e_{L}, \lambda_{L 1}, \lambda_{L 2}\right) & \text { for } \quad x<0, \\
(0,0,0,0,0) & \text { for } \quad x>0 .
\end{aligned}\right.
$$

The vacuum Riemann problem has a solution structure that is different from a conventional Riemann problem, but it can be obtained as a limit of the conventional Riemann problem, [21, 8].

We start with a brief description of the vacuum Riemann problem and Munz's tracking method and then extend the algorithm for a general EOS of the form $p=p\left(v, e, \lambda_{1}, \lambda_{2}\right)$. Figure 2a shows a Riemann problem that consists of constant left state $\mathbf{U}_{L}$, that is connected to a left-going fan, that terminates on a constant state $\mathbf{U}_{1}$ that has a particle velocity that defines a contact line in $x, t$ space. The right state $\mathbf{U}_{R}$ is connected by a right going shock to another constant state $\mathbf{U}_{2}$ whose pressure and particle velocity (but not necessarily the density) must match across the contact discontinuity to the constant state $\mathbf{U}_{1}$. Figure $2 \mathrm{~b}$, shows a left state $\mathbf{U}_{L}$, that is connected by a single (left-going) rarefaction fan that terminates on the vacuum state denoted, $\mathbf{U}_{0}$. In what follows, the velocity of the material vacuum interface (MVI) is $u_{v}$.

Next we recount the argument that the scenario shown in Figure 2a, cannot be used as a solution to the VRP, (or rather its analysis collapses the configuration to that shown in Figure 2b.) Given the configuration shown in Figure 2a, the jumps in the state across the right-going shock with speed $S$ (say) must obey the standard Rankine-Hugoniot conditions for fixed composition,

$$
\begin{aligned}
\rho_{2} u_{2}-\rho_{0} u_{0} & =S\left(\rho_{2}-\rho_{0}\right), \\
\rho_{2} u_{2}^{2}+p_{2}-\left(\rho_{0} u_{0}^{2}+p_{0}\right) & =S\left(\rho_{2} u_{2}-\rho_{0} u_{0}\right), \\
u_{2}\left(\rho_{2} E_{2}+p_{2}\right)-u_{0}\left(\rho_{0} E_{0}+p_{0}\right) & =S\left(\rho_{2} E_{2}-\rho_{0} E_{0}\right) .
\end{aligned}
$$

The state on the left side of the shock as shown is the state $\mathbf{U}_{2}$. In this case we have $\rho_{0}=0$, and this leads to the conclusion that

$$
u_{2}=u_{0}=S, \quad p_{2}=p_{0} .
$$

But if $\mathbf{U}_{R}$ is the vacuum state $\mathbf{U}_{0}$, then $p_{0}=0$, hence $p_{2}=0$. The conditions across the contact discontinuity show that $p_{1}=0$. The solution scenario shown in Figure 2. If the regurarity conditions (19) are imposed, then the zero pressure state is a zero density state. Hence both states $\mathbf{U}_{1}$ and $\mathbf{U}_{2}$ are vacuum states, now connected to a left-going rarefaction fan. Therefore the solution of VRP with vacuum right state consists of 
left rarefaction wave and the tail of the rarefaction merges at the vacuum interface as shown in the Figure $2 \mathrm{~b}$.

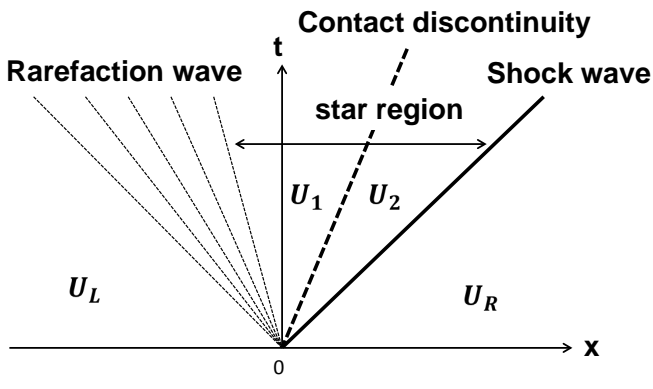

(a)

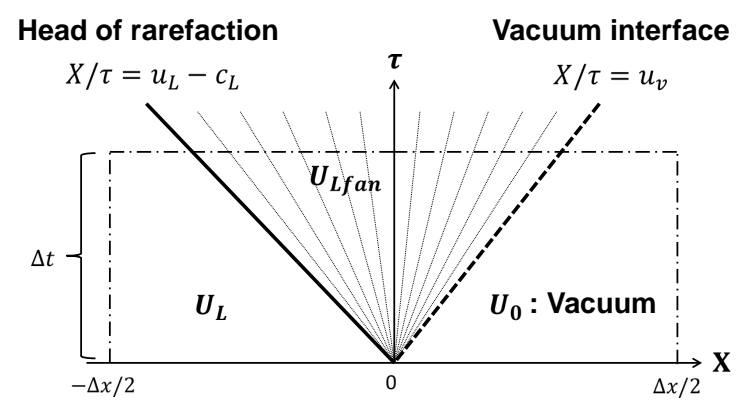

(b)

Figure 2: (a) A solution of Vacuum Riemann Problem: This solution is not possible if $\mathbf{U}_{R}$ is a vacuum state. (b) A possible solution of a Vacuum Riemann Problem.

Thus Riemann problem for vacuum right state has the left non-vacuum constant states $\mathbf{U}_{L}=\left(\rho_{L}, u_{L}, p_{L}\right)$ and the right vacuum state

$$
\mathbf{U}_{0}=\left(\rho_{0}, u_{0}, p_{0}\right) \equiv(0,0,0)
$$

where $u_{v}$ is the velocity of the material vacuum interface (MVI). One elementary wave (isentropic, rarefaction fan) connects the left non-vacuum and the right vacuum state.

\subsubsection{Numerical flux approximation}

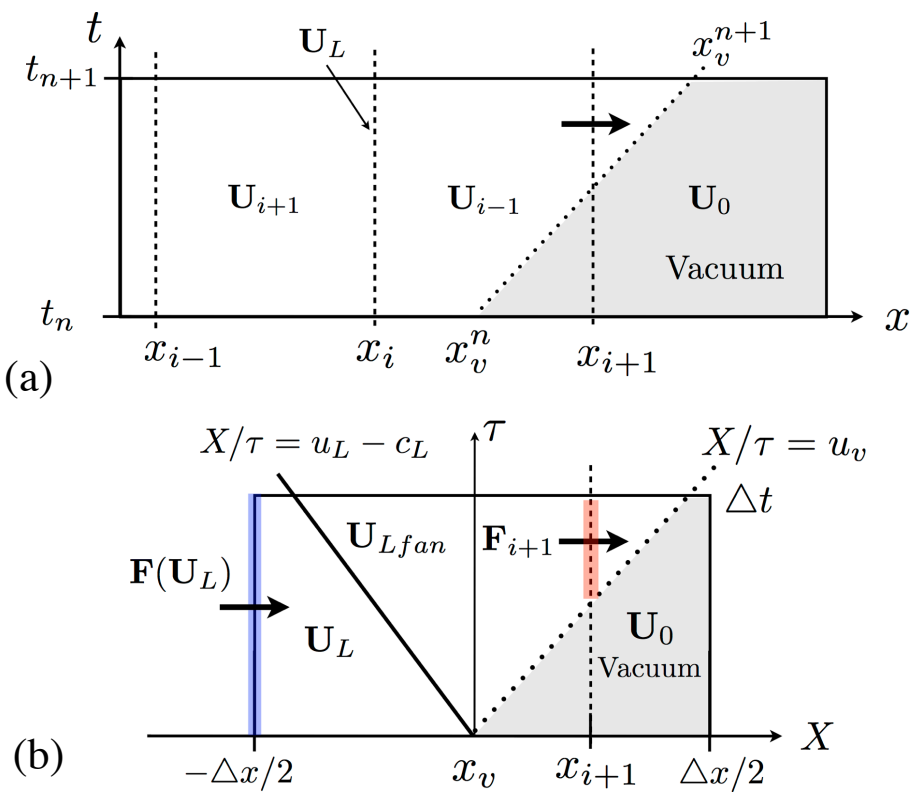

Figure 3: (a) Tracking of vacuum interface boundary in the case that the MVI moves across the cell interface $x_{i+1}$. (b) The case of non-zero flux across the grid interface $x_{i+1}$, denoted by $\mathbf{F}_{i+1}$.

Numerical approximation of gas flow near the gas-vacuum boundary can give rise to severe difficulties in the numerical scheme, associated with zero or near-zero density used in mathematical model of the 
equation of state. The Munz approximation scheme [1] computes the flux at the gas-vacuum interface and simultaneously tracks the vacuum material interface. The original algorithm was developed for the case of inert, perfect gas. We use the Munz flux approximations and extend the algorithm updates to multicomponent reacting flow uses non-ideal EOS forms that are commonly used in explosive modeling. First, we briefly describe the Munz flux formula, for completeness and to clarify the original presentation.

Let $x_{v}^{n}$ be the location of the material vacuum interface, with velocity $u_{v}^{n}$ at the current time $t=t^{n}$. The location of the MVI at time $t_{n+1}$ is estimated by the advance

$$
x_{v}^{n+1}=x_{v}^{n}+\left(t_{n+1}-t_{n}\right) u_{v}^{n} .
$$

The numerical flux $\mathbf{F}_{i+1}$ at the $(i+1)$ th cell boundary is computed based on on the estimated position of the MVI, see Figure 3a. If the MVI does not cross the cell interface at $x_{i+1}$ at at time $t_{n+1}$, such that $x_{v}^{n+1} \leq x_{i+1}$, then the interface lies in the vacuum during the time step and the flux $\mathbf{F}_{i+1}$ is zero. If the MVI does cross the cell interface at $x_{i+1}$ at at time $t_{n+1}$ (as shown), such that $x_{v}^{n+1}>x_{i+1}$ then the flux is computed by means of solving a local Vacuum Riemann Problem (VRP) centered at $x=x_{v}^{n}$. The initial data for the VRP (for a right going MVI) is

$$
\mathbf{U}(x, 0)=\left\{\begin{array}{lll}
\mathbf{U}_{L} & \text { for } & X<0 \\
\mathbf{U}_{0} & \text { for } & X>0
\end{array}\right.
$$

where $\mathbf{U}_{0}$ is the vacuum state and $\mathbf{U}_{L}$ is a non-vacuum left state.

The VRP has the vacuum state on $\mathbf{U}_{R}=\mathbf{U}_{0}$ on the right. For the left state $\mathbf{U}_{L}$, Munz proposed an average that is weighted between the cell-centered states on the left and right of the $x_{i}$ interface

$$
\mathbf{U}_{L}=\alpha \overline{\mathbf{U}}_{i+1 / 2}^{n}+(1-\alpha) \mathbf{U}_{i-1 / 2}^{n} \quad \text { with } \quad \alpha=\frac{x_{v}^{n}-x_{i}}{x_{i+1}-x_{i}} .
$$

The cell between $x_{i}$ and $x_{i+1}$, has vacuum in the interval $\left[x_{v}^{n}, x_{i+1}\right]$, with value $\overline{\mathbf{U}}_{i+1 / 2}^{n}$ in that region. The average of the non vacuum state over the entire cell is $\mathbf{U}_{i+1 / 2}^{n}$ is defined by $\overline{\mathbf{U}}_{i+1 / 2}^{n}\left(x_{v}^{n}-x_{i}\right)=\mathbf{U}_{i+1 / 2}^{n}\left(x_{i+1}-\right.$ $x_{i}$ ) such that

$$
\mathbf{U}_{L}=\mathbf{U}_{i+1 / 2}^{n}+\frac{x_{i+1}-x_{v}^{n}}{x_{i+1}-x_{i}} \mathbf{U}_{i-1 / 2}^{n}
$$

is used for the left state.

Munz' approximate solution to this VRP is

$$
\mathbf{U}(x, t)=\left\{\begin{array}{lll}
\mathbf{U}_{L} & \text { for } & X / \tau<u_{L}-c_{L} \\
\mathbf{U}_{L f a n} & \text { for } & u_{L}-c_{L}<X / \tau<u_{v} \\
\mathbf{U}_{0} & \text { for } & X / \tau>u_{v}
\end{array}\right.
$$

where all the states are constants. The constant state $\mathbf{U}_{L f a n}$ is obtained by using the integral form of the conservation law, by integrating the conservation law over the region bound by $X=-\Delta x / 2, X=u_{v} \tau$ and $\Delta t$. See Figure $3 \mathrm{~b}$ ), gives

$$
\int_{-\Delta x / 2}^{u_{v} \Delta t} \mathbf{U}(x, \Delta t) d X=\frac{\Delta x}{2} \mathbf{U}_{L}+\mathbf{F}\left(\mathbf{U}_{L}\right) \Delta t .
$$

The state $\mathbf{U}(x, \Delta t)$ has the value $\mathbf{U}_{L f a n}$ for $x \in\left[\left(u_{L}-c_{L}\right) \Delta t, u_{v} \Delta t\right]$.

The state in that fan region is approximated with an averaged value $\mathbf{U}_{L f a n}$. If one applies the integral conservation law to the region, $x \in\left[-\triangle x / 2, x_{i+1}\right]$ and $t \in\left[t_{n}, t_{n+1}\right]$ one obtains 


$$
\int_{-\Delta x / 2}^{\left(u_{L}-c_{L}\right) \Delta t} \mathbf{U}(x, \Delta t) d X+\int_{\left(u_{L}-c_{L}\right) \Delta t}^{u_{v} \Delta t} \mathbf{U}(x, \Delta t) d X=\frac{\Delta x}{2} \mathbf{U}_{L}+\mathbf{F}\left(\mathbf{U}_{L}\right) \Delta t
$$

or

$$
\left(u_{L}-c_{L}\right) \Delta t \mathbf{U}_{L}+\frac{\Delta x}{2} \mathbf{U}_{L}+\left[u_{v}-\left(u_{L}-c_{L}\right)\right] \Delta t \mathbf{U}_{L f a n}=\frac{\Delta x}{2} \mathbf{U}_{L}+\mathbf{F}\left(\mathbf{U}_{L}\right) \Delta t,
$$

which obtains the formula for $\mathbf{U}_{L f a n}$

$$
\mathbf{U}_{L f a n}=\frac{\mathbf{F}\left(\mathbf{U}_{L}\right)-\left(u_{L}-c_{L}\right) \mathbf{U}_{L}}{u_{v}-u_{L}+c_{L}} .
$$

This approximation is subject to the standard restriction that the waves generating from an interface do not interact within the cell by waves from adjacent cell interfaces, which implies the CFL condition

$$
\Delta t \leq \frac{\Delta x / 2}{S_{\max }},
$$

where $S_{\max }$ is the maximum wave velocity i.e. $\max \left[\left|u_{L}-c_{L}\right|,\left|u_{v}\right|\right]$.

The next step is to use the approximate solution (66), (70) to calculate the flux at $x_{i+1}$, when $x_{v}^{n+1}>$ $x_{i+1}$, see Figure 3a. The left non-vacuum states lie in the interval $[-\Delta x / 2,0]$ and the vacuum takes place in the shifted interval $[0, \Delta x / 2]$. Integrating the integral conservation law across the box from $\left[-\Delta x / 2, x_{i+1}-\right.$ $\left.x_{v}^{n}\right]$, and $[0, \Delta t]$, leads to

$$
\int_{-\Delta x / 2}^{x_{i+1}-x_{v}^{n}} \mathbf{U}(x, \Delta t) d X=\frac{\Delta x}{2} \mathbf{U}_{L}+\mathbf{F}\left(\mathbf{U}_{L}^{r}\right) \Delta t-\mathbf{F}_{i+1} \Delta t .
$$

This can be recast as

$$
\int_{-\Delta x / 2}^{\left(u_{L}^{r}-c_{L}^{r}\right) \Delta t} \mathbf{U}(x, \Delta t) d X+\int_{\left(u_{L}^{r}-c_{L}^{r}\right) \Delta t}^{x_{i+1}-x_{v}^{n}} \mathbf{U}(x, \Delta t) d X=\frac{\Delta x}{2} \mathbf{U}_{L}^{r}+\mathbf{F}\left(\mathbf{U}_{L}^{r}\right) \Delta t-\mathbf{F}_{i+1} \Delta t,
$$

and on the timeline $t=t^{n+1}$ we use

$$
\mathbf{U}(x, \Delta t)=\left\{\begin{array}{lll}
\mathbf{U}_{L} & \text { for } & -\Delta x / 2<X<\left(u_{L}-c_{L}\right) \Delta t \\
\mathbf{U}_{L f a n} & \text { for } & \left(u_{L}-c_{L}\right) \Delta t<X<\left(x_{i+1}-x_{v}^{n}\right)
\end{array} .\right.
$$

From the approximate solution (66), and the equation (72), we obtain

$$
\left(u_{L}-c_{L}\right) \Delta t \mathbf{U}_{L}+\frac{\Delta x}{2} \mathbf{U}_{L}+\left[x_{i+1}-x_{v}^{n}-\left(u_{L}-c_{L}\right) \Delta t\right] \mathbf{U}_{L f a n}=\frac{\Delta x}{2} \mathbf{U}_{L}+\mathbf{F}\left(\mathbf{U}_{L}\right) \Delta t-\mathbf{F}_{i+1} \Delta t .
$$

By separate consideration of the cases when the left rarefaction boundary is such that $u_{L}^{r}-c_{L}^{r}>0$ or $u_{L}^{r}-c_{L}^{r} \leq 0$, the flux $\mathbf{F}_{i+1}$ at $x_{i+1}$ computed from (75) is written concisely as

$$
\begin{gathered}
\mathbf{F}_{i+1} \quad=\mathbf{F}\left(\mathbf{U}_{L}^{r}\right)-\frac{1}{\Delta t} \min \left[\left(u_{L}^{r}-c_{L}^{r}\right) \Delta t, x_{i+1}-x_{v}^{n}\right] \mathbf{U}_{L}^{r} \\
-\frac{1}{\Delta t} \max \left[0, x_{i+1}-x_{v}^{n}-\left(u_{L}^{r}-c_{L}^{r}\right) \Delta t\right] \mathbf{U}_{L f a n}^{r} .
\end{gathered}
$$

In summary, the method updates the interface location and determines approximate fan states in the MVI adjacent cell, consistent with the integral conservation law, then updates the flux at the vacuum 
interface boundary. The tracking method is only activated when the density of left (or right) local Riemann problem has a neighboring vacuum state. If any neighboring state is not a vacuum state, the algorithm computes the flux using the general Riemann solver (in our case the Roe-solver for general equation of state). A simple criterion is required to establish a vacuum (or extremely low pressure state). In our case a density cut off is used, and for the applications discussed in the following section sets the vacuum criteria to $10^{-9} \mathrm{~g} / \mathrm{cc}$. When the ideal equation of state is used and no reaction occurs, the velocity $x_{v}$ of vacuum interface can be computed analytically as shown by Munz. However when non-ideal EOS for reactive flow is used, the computation of vacuum interface is not simple and depends on how the reaction quenches during the isentropic expansion. Furthermore an expression for the pressure in the isentropic fan cannot be obtained in closed form and numerical integration is required. We describe these essential considerations in the next section.

\subsection{Considerations and the limiting conditions near the vacuum edge for multi-component reacting flow}

In previous section, we described the basic tracking algorithm. Here we add the considerations required to employ the algorithm for a multi-component reacting flow with mechanical and thermal mixture EOS form expressed as of the form $p=p\left(v, e, \lambda_{i}\right)$ and $p=p\left(v, T, \lambda_{i}\right)$. Menikoff [22] worked the details for a complete MG EOS, which we choose to model the component EOS forms for the discussion in this section. We assume that

$$
e_{i}\left(p, v_{i}\right)=e_{i}^{s}\left(v_{i}\right)+\frac{v_{i}}{\Gamma_{i}}\left(p-p_{i}^{s}\left(v_{i}\right)\right)+e_{0 i}
$$

where we assume that $p_{i}^{s}\left(v_{i}\right)$ and $e_{i}^{s}\left(v_{i}\right)$ are specified reference functions, and in the case when an isentrope is used as a reference curve, $e_{i}^{s}$ is such that $\partial e_{i}^{s} / \partial v_{i}=-p_{i}^{s}\left(v_{i}\right)$. Both $\Gamma_{i}$ and $e_{0 i}$ are assumed constants. If the specific heat is constant and the reference curve is an isentrope, Menikoff shows that the thermal equation of state is given by

$$
p=p_{i}^{s}\left(v_{i}\right)+\frac{c_{v i} \Gamma_{i}}{v_{i}}\left[T-T_{0}\left(\frac{v_{0}}{v_{i}}\right)^{\Gamma_{i}}\right] .
$$

The volume $v_{0}$ is a reference volume. One can also work out the form for the component entropies, to obtain

$$
s_{i}\left(p, v_{i}\right)=s_{0 i}+c_{v i} \ln \left[1+\frac{v_{0}}{\Gamma_{i} c_{v i} T_{0}}\left[p-p_{i}^{s}\left(v_{i}\right)\right]\left(\frac{v_{i}}{v_{0}}\right)^{1+\Gamma_{i}}\right] .
$$

The mixture energy and volume and entropy are given by

$$
e=\sum_{i} e_{i} \lambda_{i}, \quad v=\sum_{i} v_{i} \lambda_{i} \quad \text { and } \quad s=\sum_{i} s_{i} \lambda_{i}
$$

In the region near the vacuum edge the flow is highly rarefied, and nearly collisionless so it is reasonable to assume that the reaction rates are zero (as is the basic premise of the TOFMS experiments), and hence isentropic. The task of working out the structure of the flow in the fan is the task of finding the isentrope in the vacuum fan solution. We briefly discuss the nature of that calculation using these non-ideal EOS forms, without further assumption, except that of isentropic expansion.

The analysis of the left rarefaction fan at the right edge of the domain then requires an expression for the $p, v$ isentropes for the mixture. In turn this requires finding expressions (or numerically calculating) the values for the partial volumes $v_{i}(p, v)$ of components in the mixture. The mixture entropy is then given by

$$
s=\sum_{i} s\left(v_{i}(p, v), p\right) \lambda_{i}
$$

and is found as a function of $p, v$ and $\lambda_{i}$, in general. The determination of $v_{i}(p, v)$ depends on the forms of the mixture closure. If the mixture closure uses the PT-EQB, an iterative numerical solution is required 
even for a simplified version of the MG form. But that can always be done in principle, as the formulation is complete. The sound speed squared on the isentrope is given by $c^{2}=\partial p /\left.\partial \rho\right|_{s}$, and can be calculated

$$
c^{2}=v^{2}\left(p+\left.\frac{\partial e}{\partial v}\right|_{p, \lambda_{i}}\right) /\left.\frac{\partial e}{\partial p}\right|_{v, \lambda_{i}}
$$

to obtain $c^{2}\left(p, v, \lambda_{i}\right)$. The constant entropy in the left state $s_{L}$ (say) can be used in (81) to generate the $p, v$ isentrope that applies in the rarefaction of the form $s_{L}=s\left(p, v, \lambda_{i}\right)$. In turn this can be used to construct the solution and compute the MVI velocity.

An equivalent and computationally simple procedure is available by a directly integrating states through the fan as follows. From the consideration of the Generalized Riemann Invariants for the system (46) for the rarefaction wave $\mu_{1}=u-c$ we have

$$
d \rho=\frac{d(\rho u)}{u-c}=\frac{d(\rho e)}{H-u c} .
$$

The first equality in equation (83) gives a formula for the MVI velocity, $u_{v}$ where $\rho_{R}=0$ and $u_{R}=u_{v}$ as shown in Figure 2b.

$$
u_{v}=u_{L}+\int_{0}^{\rho_{L}} \frac{c}{\rho} d \rho .
$$

The second equality from equation (83), obtains the isentropic change of the internal energy in the isentropic expansion and is written as

$$
d e=\frac{p}{\rho^{2}} d \rho .
$$

This equation can be used to derive a differential equation for $p\left(\rho, \lambda_{i}\right)$. As an example, consider the equation of state defined by using the JWL EOS forms for two components as defined by equation (29). Using the form (34) the energy can be written as

$$
e(p, v, \lambda)=A+G p
$$

where

$$
A\left(v_{R}, v_{p}, \lambda\right)=(1-\lambda)\left(e_{R}^{s}\left(v_{R}\right)-\frac{v_{R}}{w_{R}} p_{R}^{s}\left(v_{R}\right)\right)+\lambda\left(e_{P}^{s}\left(v_{P}\right)-\frac{v_{P}}{w_{P}} p_{P}^{s}\left(v_{P}\right)\right)
$$

and

$$
G\left(v_{R}, v_{p}, \lambda\right)=\lambda \frac{v_{P}}{\omega_{P}}+(1-\lambda) \frac{v_{R}}{\omega_{R}} .
$$

The closure condition is used to determine the component specific volumes $v_{P}$ and $v_{R}$. The PT-EQB condition for the thermal EOS form is

$$
T \equiv T_{R}\left(v_{R}, p\right)=T_{P}\left(v_{P}, p\right),
$$

where $T_{R}$ and $T_{P}$ are obtained from the thermal EOS for the components. With $\Phi=v_{P} / v_{R}$, and equation (30) leads to

$$
v_{R}=\frac{v}{1-\lambda+\lambda \Phi}, \quad v_{P}=\frac{v \Phi}{1-\lambda+\lambda \Phi}
$$

Equation (89) determines a value of $\Phi(p, v)$ for fixed $\lambda$. Given a value $\lambda$, the functions $A$ and $G$ are functions of $v$ and $p$. Then if we substitute equation (86) in (85) one obtains a differential equation for the pressure as a function of density for fixed composition, that holds in the rarefaction wave 


$$
\frac{d p}{d \rho}=\frac{1}{G+\frac{\partial A}{\partial p}+p \frac{\partial G}{\partial p}}\left[\left\{\frac{1}{\rho^{2}}-\left(\frac{\partial G}{\partial \rho}\right)\right\} p-\frac{\partial A}{\partial \rho}\right], \quad \text { subject to } \quad p\left(\rho_{L}\right)=p_{L} .
$$

Note that the $c^{2}=d p / d \rho$ on the isentrope. The interface velocity is then computed from (84) as

$$
u_{I}=u_{L}+\int_{0}^{\rho_{L}} \frac{1}{\rho} \sqrt{\frac{1}{G+\frac{\partial A}{\partial p}+p \frac{\partial G}{\partial p}}\left[\left\{\frac{1}{\rho^{2}}-\left(\frac{\partial G}{\partial \rho}\right)\right\} p-\frac{\partial A}{\partial \rho}\right]} d \rho .
$$

The pressure $p$ in the integrand of equation (92) is the solution of the ODE (91).

Generally the ODE for $p$ and the integral for $u_{v}$ must be computed numerically. However if the simpler P-EQB closure is used to compute the component volumes as in Stewart et al. in [23], then $\Phi$ does not depend on pressure and the ODE (91) simplifies to

$$
\frac{d p}{d \rho}=\frac{1}{G}\left[\left\{\frac{1}{\rho^{2}}-\left(\frac{d G}{d \rho}\right)\right\} p-\frac{d A}{d \rho}\right], \quad \text { subject to } \quad p\left(\rho_{L}\right)=p_{L},
$$

and can be integrated analytically to obtain the solution for $p$

$$
p(\rho)=p_{1}\left(\frac{\rho}{\rho_{1}}\right)^{\Gamma+1}+\Gamma \rho^{\Gamma+1}\left(A\left(\rho_{1}\right) \rho_{1}^{-\Gamma}-A(\rho) \rho^{-\Gamma}+\Gamma \int_{\rho}^{\rho_{1}} A \rho^{-\Gamma-1} d \rho\right) .
$$

where

$$
\Gamma=(1-\lambda+\lambda \Phi) /\left(\frac{\lambda}{\omega_{p}}+\frac{(1-\lambda) \Phi}{\omega_{R}}\right)
$$

so that $G=\frac{v}{\Gamma}$.

At the limit of zero density, one must check the Liu-Smoller conditions (19) to make sure that the pressure and sound speed vanish as the density vanishes. The solution satisfies the end condition $p(0)=0$. The derivative is given by

$$
\frac{d p}{d \rho}=(\Gamma+1)\left(\frac{p_{L}}{\rho_{L}^{\Gamma+1}}+\Gamma \frac{A\left(\rho_{L}\right)}{\rho_{L}^{\Gamma}}+\Gamma^{2} \int_{\rho}^{\rho_{L}} \frac{A}{\rho^{\Gamma+1}} d \rho\right) \rho^{\Gamma}-\Gamma \rho \frac{d A}{d \rho}-\Gamma(\Gamma+1) A(\rho),
$$

and the solution satisfies the Liu-Smoller conditions (19) if $A=o\left(\rho^{\Gamma+1}\right)$ and $A^{\prime}=o\left(\rho^{\Gamma+1}\right)$ as $\rho \rightarrow 0$.

Physical consideration of the near collision limit of a vacuum would admit the reasonable approximation that each EOS for each gaseous component of the mixture limits to an ideal gas form. For each component in the limit of infinite volume, or zero density we would require that

$$
e_{i}^{s}\left(v_{i}\right) \rightarrow 0, \quad p_{i}^{s}\left(v_{i}\right) \sim \frac{c_{v i} \Gamma_{i}}{v_{i}}\left[T_{0}\left(\frac{v_{0}}{v_{i}}\right)^{\Gamma_{i}}\right] \rightarrow 0 \quad \text { as } \quad \rho_{i}=1 / v_{i} \rightarrow 0,
$$

so that in the limit of low densities the ideal EOS forms apply

$$
e_{i}\left(p, v_{i}\right) \sim \frac{p v_{i}}{\left(\gamma_{i}-1\right)}+e_{0 i}, \quad \text { and } \quad p \sim \frac{R_{i} T}{v_{i}} .
$$

where $c_{v i} \Gamma_{i} \rightarrow R_{i}$ (the ideal gas constant weighted by the molecular weight of the component) and $\Gamma_{i} \rightarrow$ $\gamma_{i}-1$. One can view these additional requirements as a modeling statement that reflects the correct physics or simply a physically-based regularization for the model that is used only near the MVI. In either case the additional requirement that each gaseous component EOS form limits to ideal EOS form when PT-EQB is used as the mixture closure model, will guarantee that the Liu-Smoller conditions are met which we show next.

For the conditions near the MVI, the state will be such that the EOS forms of the components of the mixture limit to the ideal EOS forms. For fixed pressure and temperature, we can take the expression for 
the partial volumes and sum them over the components to obtain

$$
v=\sum_{i} v_{i} \lambda_{i}=\left(\sum_{i} R_{i} \lambda_{i}\right) \frac{T}{p}=\frac{R T}{p}
$$

where $R=\sum_{i} R_{i} \lambda_{i}$ is the mass weight ideal gas constant. Then specific energy of the multi-component mixture limits to

$$
e \sim \frac{p v}{\gamma-1}+\sum_{i} e_{i 0} \lambda_{i}, \quad \text { with } \quad \frac{1}{\gamma-1} \equiv\left(\sum_{i} \frac{\lambda_{i}}{\left(\gamma_{i}-1\right)} \frac{R_{i}}{R}\right)
$$

and where we have used $T \sim p v / R$ and identified, and $\gamma$ is a function of the composition. Then the standard formula for sound speed squared

$$
c^{2}=\gamma p v
$$

and the isentrope in the fan expansion is given by

$$
\frac{p}{\rho^{\gamma}}=\kappa
$$

where the constant $\kappa$ is also a function of the frozen composition, which determines $\gamma$. If these limiting forms for the EOS are used then the Liu-Smoller conditions are satisfied since $p / \rho^{\gamma}=\kappa=p_{L} /\left(\rho_{L}^{\gamma}\right)>0$ and $c^{2}=\gamma p / \rho$ implies $c=\sqrt{\gamma \kappa} \rho^{(\gamma-1) / 2}$ with the properties that

$$
c(\rho)>0 \text { for } \rho>0 \text { and } c(0)=0
$$

and

$$
\int_{0}^{\rho_{L}} \frac{c}{\rho} d \rho=\frac{2 \sqrt{\gamma \kappa}}{\gamma-1} \rho_{L}^{(\gamma-1) / 2} \quad \text { bounded } .
$$

It is important to compute the integral (92) carefully, since there is often an abrupt change in the states through the rarefaction, especially when a shock wave first hits the MVI. To avoid singularity occurring from lower limit ofthe integrand in (92), we always split the integration into two pieces as shown in Figure 4. We define a cut-off density $\epsilon$ that is small enough to ensure that the ideal gas limiting form is a good physical approximation to the flow, and write the integral

$$
\int_{0}^{\rho_{L}} \frac{c}{\rho} d \rho=\int_{0}^{\epsilon} \frac{c}{\rho} d \rho+\int_{\epsilon}^{\rho_{L}} \frac{c}{\rho} d \rho .
$$

For a given specified value of $\epsilon$, with $0<\epsilon<<1$, the first term of (105) is evaluated with equation (104)

$$
\int_{0}^{\epsilon} \frac{c}{\rho} d \rho=\frac{2 \sqrt{\gamma \kappa}}{\gamma-1} \rho_{L}^{(\gamma-1) / 2},
$$

and the second integral is computed numerically using the Romberg's method [24].

As an example, the JWL EOS forms are MG forms that are commonly used to model explosives and they have the property that they limit to ideal EOS forms as the component densities vanish. A JWL EOS for a component takes the form

$$
\begin{gathered}
p_{i}^{s}=A_{i} e^{-R_{1 i}\left(v_{i} / v_{0}\right)}+B_{i} e^{-R_{2 i}\left(v_{i} / v_{0}\right)}+\frac{R_{i} T_{0}}{v_{0}}\left(\frac{v_{0}}{v_{i}}\right)^{\gamma_{i}} \\
e_{i}^{s}=\frac{A_{i}}{R_{1 i}} e^{-R_{1 i}\left(v_{i} / v_{0}\right)}+\frac{B_{i}}{R_{2 i}} e^{-R_{2 i}\left(v_{i} / v_{0}\right)}+\frac{R_{i} T_{0}}{\left(\gamma_{i}-1\right)}\left(\frac{v_{0}}{v_{i}}\right)^{\left(\gamma_{i}-1\right)}
\end{gathered}
$$




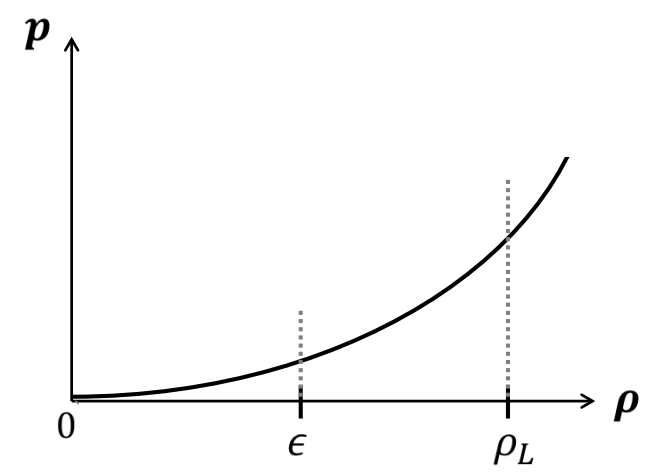

Figure 4: Two ranges of integration of equation (105).

and as $\rho_{i}=1 / v_{i} \rightarrow 0$, it follows that (97) holds. Thus the JWL EOS behaves like the ideal gas near vacuum region or density is very small, which is a feature built into the JWL EOS calibration.

If the non-ideal EOS forms for the components do limit to the ideal EOS forms in the limit of vanishingly small density, then the classic fan analysis will hold at the extreme edge of the fan. Even then we make the observation that the computed MVI velocity can be very sensitive to the limiting values of the flow near the fan when the reaction ceases, since the composition of the frozen/quenched products determines the mass fraction weighted value of $\gamma$ of the mixture.

Close to the MVI assume that the expansion to vacuum occurs by a left-going isentropic fan seeded by a constant left state that lies in a region a nonzero density, but where the ideal EOS forms are applicable. This would be a point close to the MVI edge, but to the left of it (say). Then the particle velocity and speed relation hold between the defined left state and any state to the right toward the MVI, i.e.

$$
u+\frac{2 c}{\gamma-1}=u_{L}+\frac{2 c_{L}}{\gamma-1} .
$$

Equation (108) can be used to illustrate why the tracking of vacuum interface numerically can be very difficult if done without knowledge of the analytical character of the fan solution.

Since the fan boundary at the MVI is characteristic, then $u_{v}=X / \tau$, and this is consistent with setting $u=u_{v}$ and $\rho=c=0$ at the vacuum interface. Then (108) becomes

$$
u_{v}=X / \tau=u_{L}+\frac{2 c_{L}}{(\gamma-1)} .
$$

Equation (109) could be used to compute $u_{v}$ near the interface, but since the Liu-Smoller requirement insists that in a (computationally) small neighbor of the interface $c_{L} \rightarrow 0$, under resolution, then this relation is simply the identity $u_{v}=X / \tau=u_{L}$.

But now consider equation (108) in the same small neighborhood, and consider another neighboring state to the right of the same left state in the left fan. We identify this state with a vacuum cut-off density $d$ (say) to designate being close enough to the MVI. Replace $u_{v}=X / \tau$, as before, but estimate the sound speed which is a function of $\rho$ at the vacuum cut off as

$$
c(d)=c_{L}\left(\frac{d}{\rho_{L}}\right)^{(\gamma-1) / 2} .
$$

in which case (108) becomes

$$
u_{v}=u_{L}+\frac{2 c_{L}}{\gamma-1}-\frac{2 c_{L}}{\gamma-1}\left(\frac{d}{\rho_{L}}\right)^{(\gamma-1) / 2} .
$$


There are clearly many ways to go wrong if the local approximations near the vacuum interface are not accurate, inconsistent with the Liu-Smoller conditions or inconsistent with the fan structure. The greatest sensitivity in the formulas are illustrated by the dependence on the local value of $\gamma$. As $\gamma \rightarrow 1$ the interface velocity function (111) becomes singular, and thus is very sensitive to $\gamma$. Figure 5 shows the plot of velocity $u_{v}(d)$ of equation (111) as a function of density $d$ for different values of $\gamma$; i.e $\gamma=1.2,1.4,1.8$, and 2.0. The left state variable $\rho_{L}, p_{L}$ and $u_{L}$ shown in equation (111) was set to actual computational values at time $t=1.5 \mu \mathrm{sec}$ used in the PETN problem in Section 4.2. As shown in the figure, the interface velocity increases rapidly and the slope of $u_{v}(d)$ becomes very large as $\gamma$ decreases toward one. Also it is clear from the dependence of $\rho_{L}$ in the denominator of equation (111) that if the monotonically decreasing ratio of $\left(d / \rho_{L}\right)$ is not maintained correctly in the approximation scheme, even for fixed $c_{L}$ that the prediction of the interface velocity can be made highly inaccurate. Since $\gamma$ is a function of the mass fraction as seen in equation (100), it is dependent on the composition of the products near the vacuum edge. Thus the details of the kinetic rate law and how the reactions quench, does matter in the calculation of the MVI velocity $u_{v}$.

The conclusions from the simple considerations above are independent of the grid resolution. The vacuum tracking algorithm uses the fan solution that maintains the correct limiting forms, derived from the EOS forms of the underlying model as the vacuum state is approached. The requirement that the EOS forms limit to the ideal EOS forms and that the reaction stops, is easy to implement and is physically consistent. The JWL EOS form is an example of a non-ideal form that has the correct properties and the Liu-Smoller condition are automatically satisfied. Any vacuum tracking scheme that would work similarly, must employ consideration of the EOS forms used for the mixture in the vicinity of the vacuum, otherwise large and likely catastrophic inaccuracies will occur that will destroy any simulation that has a vacuum or extremely low pressure regions. In the next section, we demonstrate application of the algorithms with examples that explicitly uses ideal and nonideal EOS forms for the mixture of reactants and their products.

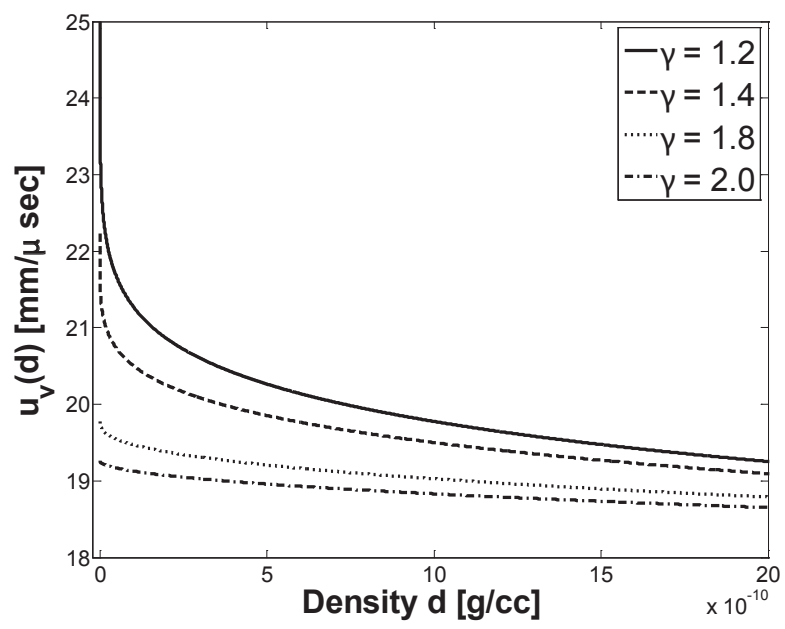

Figure 5: The plot of interface velocity function $u_{v}(d)$ shown in equation (111) with $\rho_{L}=3.0 e-8[\mathrm{~g} / \mathrm{cc}], p_{L}=3.6 e-8[G P a]$, $u_{L}=16.5[\mathrm{~mm} / \mu \mathrm{sec}]$.

\section{Examples and Applications}

In this section, we demonstrate the applicability of our algorithm through two representative numerical simulations. The first example uses the ideal equation of state. The second example is representative of the TOFMS experiments which uses the non ideal JWL EOS forms for reactants and products. 


\subsection{Example 1: An explosive modeled by the ideal gas EOS form whose products expand into a vacuum}

In many theoretical studies of high explosives (that are condensed solids at their initial state), the flows are modeled with the ideal EOS forms for a mixture of reactants and products. The reader is referred to Fickett and Davis [12]. We used values listed in their text for this example ([12], Chapter 2, C2, p.46). The EOS is given by

$$
e(p, v, \lambda)=\frac{p v}{\gamma-1}-Q \lambda .
$$

The density in the unreacted explosive is assumed to be $\rho_{0}=1.6 \mathrm{~g} / \mathrm{cc}$. For the choices $\gamma=3.0$, and $Q=4.5156 \mathrm{MJ} / \mathrm{kg}$, the Chapman-Jouguet (CJ) detonation velocity and pressure are $D_{C J}=8.5 \mathrm{~mm} / \mu \mathrm{sec}$ and $p_{C J}=28.9 \mathrm{GPa}$, respectively.

The computational domain is taken to be $50 \mathrm{~mm}$ long. The boundary at $x=0$ is an outflow boundary that enforces zero gradients. The material in the region $0 \leq x<20 \mathrm{~mm}$ is assumed to be a uniform, completely reacted, CJ detonation state with the state vector as $\mathbf{U}_{C J}=(\rho, u, p, \lambda)=\left(\rho_{C J}, u_{C J}, p_{C J}, 1\right)$. In the strong shock approximation, the CJ states are $\left.\rho_{C J}=(\gamma+1) / \gamma\right) \rho_{0}, u_{C}=D_{C J} /(\gamma+1)$, and $p_{C J}=\rho_{0} D_{C J}^{2} /(\gamma+1)$. The material between $20 \leq x<30 \mathrm{~mm}$ is assumed to be at a uniform, completely unreacted, motionless state at an initial density $\rho_{0}$, but at zero pressure with $\mathbf{U}_{H E}=(\rho, u, p, \lambda)=\left(\rho_{0}, 0,0,0\right)$. The region to the right of $x=30 \mathrm{~mm}$ is assumed to be the vacuum state $\mathbf{U}_{0}=(0,0,0,0)$. Thus the initial values are given by

$$
\mathbf{U}(x)=\left\{\begin{array}{lll}
\mathbf{U}_{C J}(\text { reacted } \mathrm{CJ}) & \text { if } \quad x<20 \\
\mathbf{U}_{H E}(\text { unreacted HE) } & \text { if } \quad 20 \leq x<30 \\
\mathbf{U}_{0} \text { (vacuum) } & & x>30
\end{array}\right.
$$

and listed in Table 1 . The reaction rate in the explosive is taken as

$$
\dot{\lambda}=(5.0)(1-\lambda)^{0.5}\left(\frac{p}{p_{C J}}\right)^{3} \mu \sec ^{-1},
$$

\begin{tabular}{|c|c|c|c|}
\hline State & Reacted CJ state & Uneacted CJ state & Vacuum state \\
\hline $\mathbf{U}$ & $0<x<20$ & $20<x<30$ & $20<x<30$ \\
\hline$\rho$ & $2.133 \mathrm{~g} / \mathrm{cc}$ & $1.6 \mathrm{~g} / \mathrm{cc}$ & 0 \\
\hline $\mathrm{u}$ & $2.125 \mathrm{~mm} / \mu \mathrm{sec}$ & 0 & 0 \\
\hline $\mathrm{p}$ & $28.9 \mathrm{GPa}$ & 0 & 0 \\
\hline
\end{tabular}

Table 1: Initial conditions for the simulation in Example 1.

The initial high pressure, density, particle velocity state, $\mathbf{U}_{C J}$ introduces a shock into the unreacted HE at the $x=20 \mathrm{~mm}$ interface and initiates a detonation wave, that builds up and runs in the unreacted HE towards the vacuum interface initially at $x=30 \mathrm{~mm}$. Since the pressure in the unreacted HE is zero, there is no initial expansion into the vacuum, so the MVI at $x=30 \mathrm{~mm}$ is motionless until the detonation shock hits the interface and the detonation products start to vent into the vacuum region. The initiated detonation becomes nearly steady, with a von Neumann spike shock pressure of about 55 GPa pressure at approximately $t=1.3 \mu \mathrm{sec}$. At that time it encounters the previously static vacuum interface. After the detonation shock and the following flow in the detonation reaction zone hits the vacuum interface at $x=30$ $\mathrm{mm}$, the detonation shock disappears and a rarefaction wave is sent backwards to the left into the gas flow. Since reaction rate in the explosive is a function of the pressure, the reaction rate in the fan region drops as the pressure drops. Figures 6 and 7 show the state variables at different times and show the formation of a detonation, followed by the expansion of products as they vent into the vacuum region. The simulation uses 5000 grid points for the entire domain, and exhibits cleanly defined, well-resolved features and a sharply defined MVI motion. 
(a)

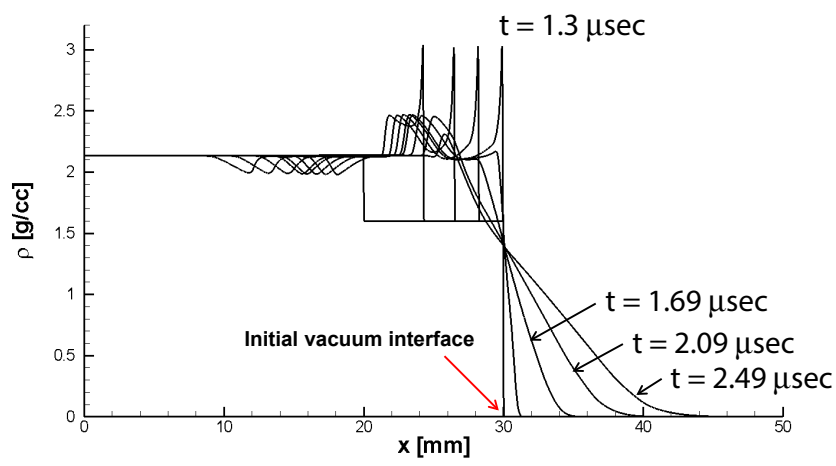

(b)

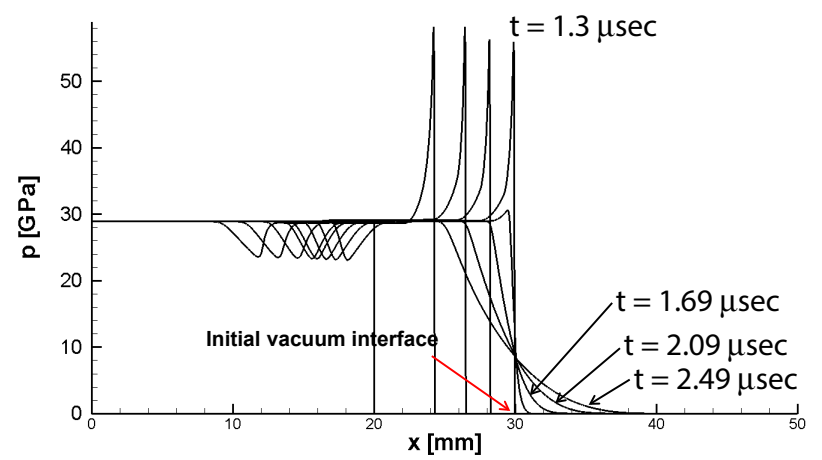

(c)

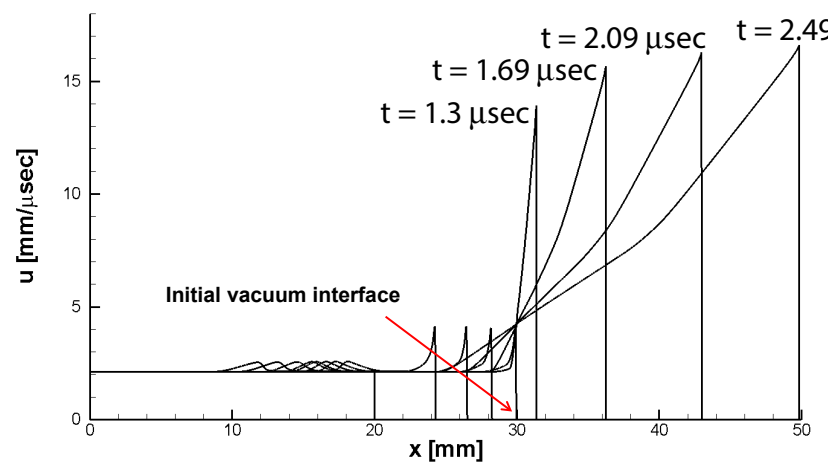

(d)

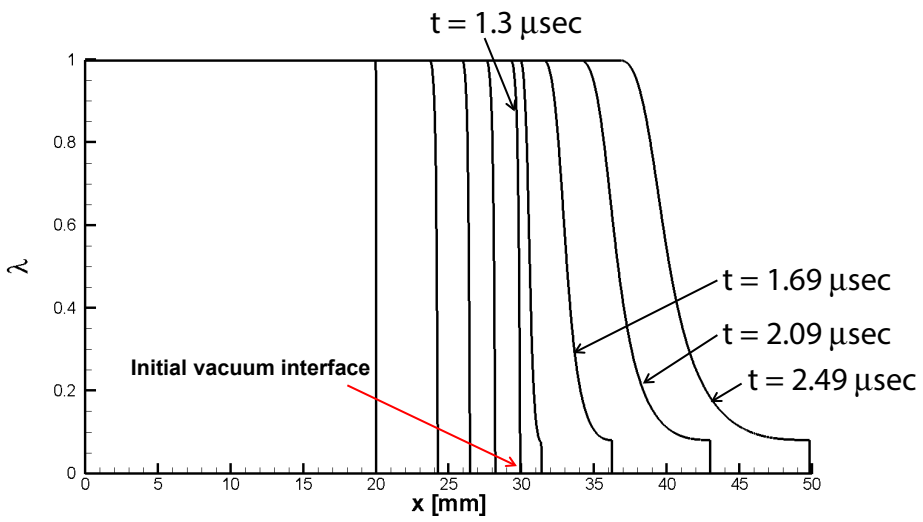

Figure 6: Sequence showing the detonation hitting the MVI and the expansion of products into the vacuum region: (a) Density, (b) Pressure, (c) Velocity, (d) Reaction Progress . 


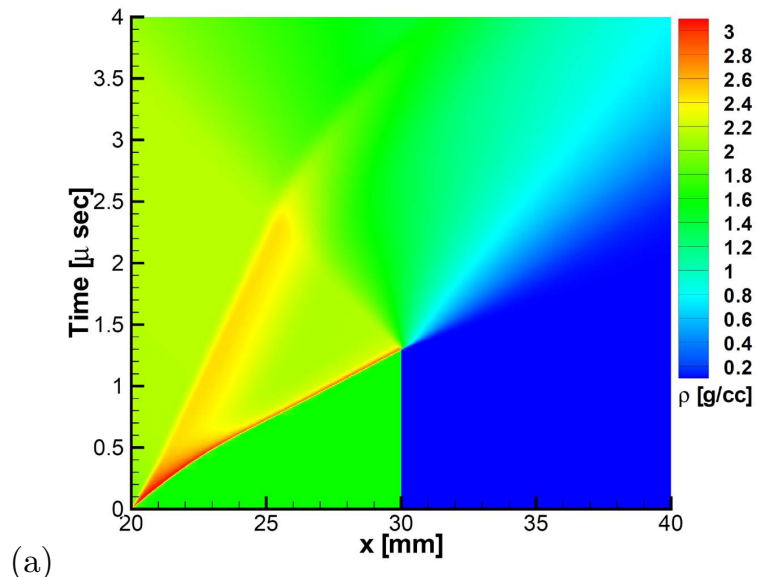

(b)
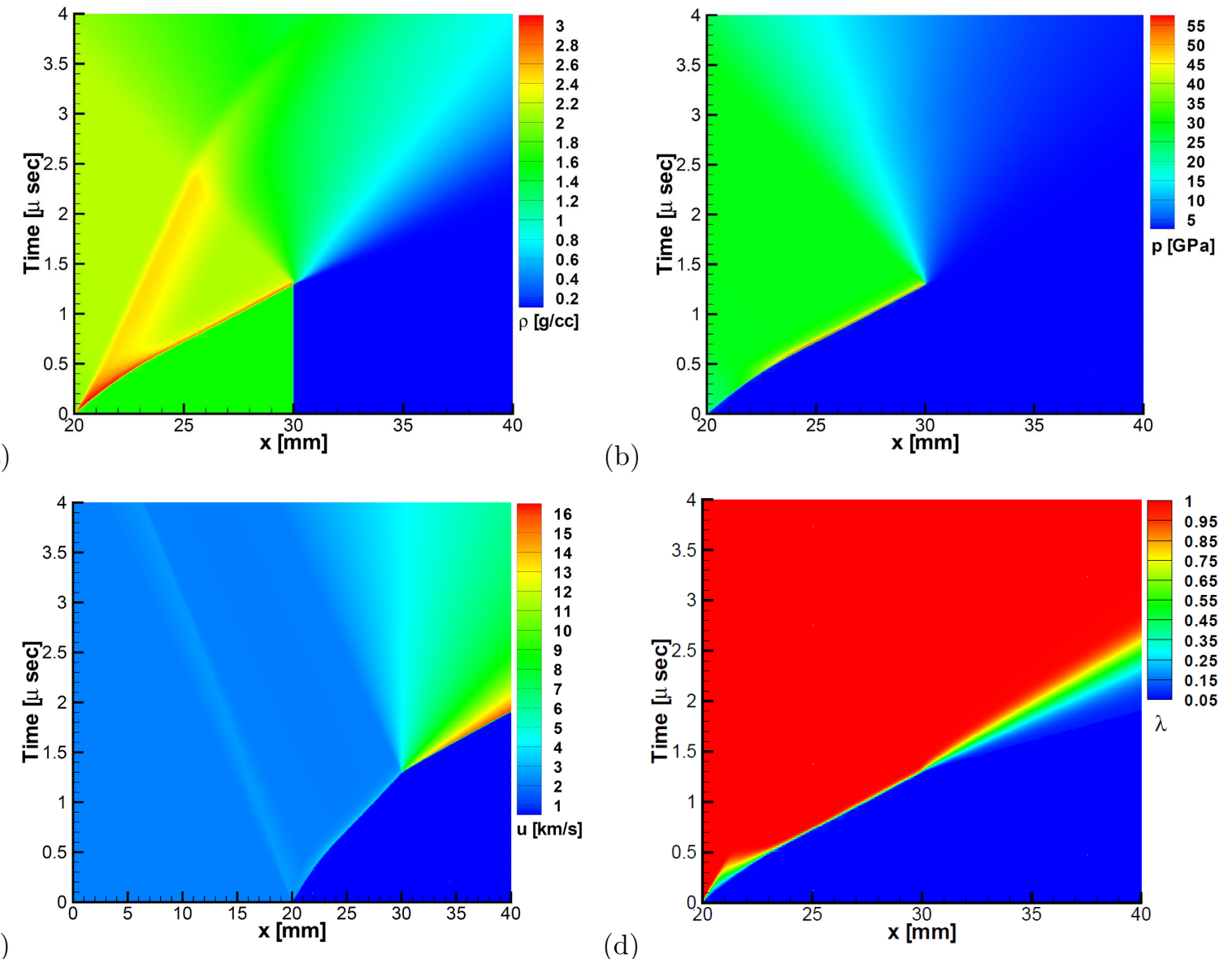

Figure 7: Space time contour plots that show the same sequence as Figure 6 showing the detonation hitting the MVI and the expansion of products into the vacuum region: (a) Density, (b) Pressure, (c) Velocity, (d) Reaction Progress field in space and time domain .

\subsection{Example 2: Detonation of pentaerythritol tetranitrate (PETN) whose products vent to vacuum}

Next we describe a simulation of the expansion of PETN products into a vacuum, that represents a facsimile of TOFMS experiments. The JWL EOS parameters for reactants and products are chosen to match experimental data obtained from macroscopic detonation experiments on PETN, [26]. These experiments include those that determine: a) the unreacted shock Hugoniot or $U_{p}-U_{s}$ relation, b) the overdriven detonation shock Hugoniot for the products, and c) the products expansion experiments that determine constants for the products JWL EOS, [27, 25, 28, 26]. The reaction rate parameters are determined from shock initiation experiments that determine the distance and time to detonation as a function of the shock input pressure into the sample. The reader can find details on how the equation of state and rate law parameters are fit to explosives in recent review papers, [29], [30].

We use model parameters reported by Tarver et al. in [26]. The calibrated parameters of the JWL EOS forms for the reactant and product are given in Table 2. The reference density is given by $\rho_{0}=1.778$ $\mathrm{gm} / \mathrm{cm}^{3}$. The JWL product equation of state parameters determines the CJ states which are found to be

$$
D_{C J}=8.23 \mathrm{~mm} / \mu \mathrm{sec}, v_{C J}=0.42[\mathrm{cc} / \mathrm{gm}], p_{C J}=31.5 \mathrm{GPa}
$$

The distance to detonation for full density PETN is reported as 


\begin{tabular}{c|ccccccc} 
& $A_{i}[\mathrm{GPa}]$ & $B_{i}[\mathrm{GPa}]$ & $C_{i}$ & $w_{i}$ & $R 1_{i}$ & $R 2_{i}$ & $e_{0 i}[\mathrm{KJ} / \mathrm{g}]$ \\
\hline Product JWL (i=R) & 1032.158 & 90.57 & 0.0 & 0.57 & 6 & 2.6 & 6.074 \\
Reactant JWL (i=P) & 1280.0 & -27.058 & 0.0 & 0.6 & 6 & 2 & 0.0
\end{tabular}

Table 2: The calibrated parameters of JWL EOS of PETN.

$$
\log _{10} p=a-b \log _{10} x^{*}
$$

where $p[\mathrm{GPa}]$ is the shock input pressure and $x^{*}[\mathrm{~mm}]$ is observed distance to detonation from the explosive edge, and parameters $a=1.1767[G P a], b=0.2432$. We used a simple, pressure dependent reaction rate model with rate parameters chosen so that our PETN model reproduces the reported the run to detonation data, (116). The rate is given by

$$
\frac{d \lambda}{d t}=80(1-\lambda)^{0.5}\left(\frac{p}{p_{C J}}\right)^{5} \mu \sec ^{-1} .
$$

\begin{tabular}{c|cccc}
\hline material & $p[\mathrm{Gpa}]$ & $\rho\left[\mathrm{g} / \mathrm{cm}^{3}\right]$ & $u[\mathrm{~mm} / \mu \mathrm{sec}]$ & range \\
\hline piston & 0.0 & 2.14 & $u_{\text {in }}$ & $x \leq x_{I}$ \\
PETN & 0.0 & 1.778 & 0.0 & $x_{I} \leq x \leq x_{v}$ \\
Vacuum & 0 & 0 & 0.0 & $x>x_{v}$ \\
\hline
\end{tabular}

Table 3: Initial data for impact simulation Example 2.

Figure 1 shows a schematic of the setup for a simulation of motionless PETN that has vacuum on the right side, and is struck by a impact piston on the right side. Initial states $(p, \rho, \lambda)$ at initial time $t=0$ are divided into three regions and are shown in Table 3. Through impact on the left side, a shock is introduced into the unreacted material. If the shock is strong enough and the sample is thick enough, then a steady state detonation forms in the PETN sample in the region $x_{I}<x<x_{v}$ before the detonation shock strikes the vacuum interface, after which the venting at the interface occurs. In PETN, the steady CJ detonation structure has a reaction complements of approximately $99 \%$ in $0.02 \mathrm{~mm}$, and the final $1 \%$ of the energy that relaxes to the CJ state, is released in a reaction zone tail that is approximately $0.2 \mathrm{~mm}$. Therefore the computation requires high resolution, and the spatial grid size must be no more than one micrometer with approximately 20 points in the rapidly changing detonation shock structure. Note that a multi-material solver, described in [31], was used to properly model the flyer impact (of a different material, specifically a plastic KEL-F) and transmission of the impact shock into the PETN sample. But the interior algorithms and the algorithms near the MVI are identical to what is discussed in this paper.

Experimental detection of product species flying through vacuum depends on many factors, that include the thickness of sample and how the sample is shock initiated. A premise of the experiment is that a steady detonation is established by the time the detonation shock hits the vacuum interface and the reactants are quenched by the subsequent rarefaction. When the PETN sample is initiated with an input shock strength of $5 \mathrm{GPa}$ in pressure, matched to the experimental pop-plot shows a steady state detonation is established in about $0.16 \mu \mathrm{sec}$ at around $0.5 \mathrm{~mm}$ away from the edge of the sample. In this scenario, any sample thicker than $0.5 \mathrm{~mm}$ is sufficiently thick to observe steady state detonation. Once the steady state detonation on PETN hits the vacuum interface and the vented products expand at a very high speed, faster than 16 $\mathrm{mm} / \mu \mathrm{sec}$.

Next we discuss a set of cases in which the thickness of the sample, i.e. $x_{v}-x_{I}$ and the impactor velocity $u_{\text {impactor }}$ (i.e. related to the input shock pressure) are varied. In each case the density $\rho$, pressure $p$ and reaction progress $\lambda$ are sampled at a designated point far away from the original vacuum interface locations 
and the time histories are recorded, similar to what would be recorded at a sampling probe at a fixed point in space in the TOFMS experiment. The three different cases are

- Case I: $\quad x_{v}-x_{I}=3.0 \mathrm{~mm}, \quad u_{\text {impactor }}=2.963 \mathrm{~mm} / \mu \mathrm{sec}$

- Case II: $x_{v}-x_{I}=1.0 \mathrm{~mm}, \quad u_{\text {impactor }}=2.963 \mathrm{~mm} / \mu \mathrm{sec}$

- Case III: $x_{v}-x_{I}=3.0 \mathrm{~mm}, \quad u_{\text {impactor }}=2.563 \mathrm{~mm} / \mu \mathrm{sec}$

Figure 8 shows the locations of the impactor, PETN (HE sample) and vacuum and probe locations where measurements are recorded. The probe locations are set to $x=100,200,300,400,500,600$ [mm] and $x=680[\mathrm{~mm}]$ measured from the initial vacuum interface.

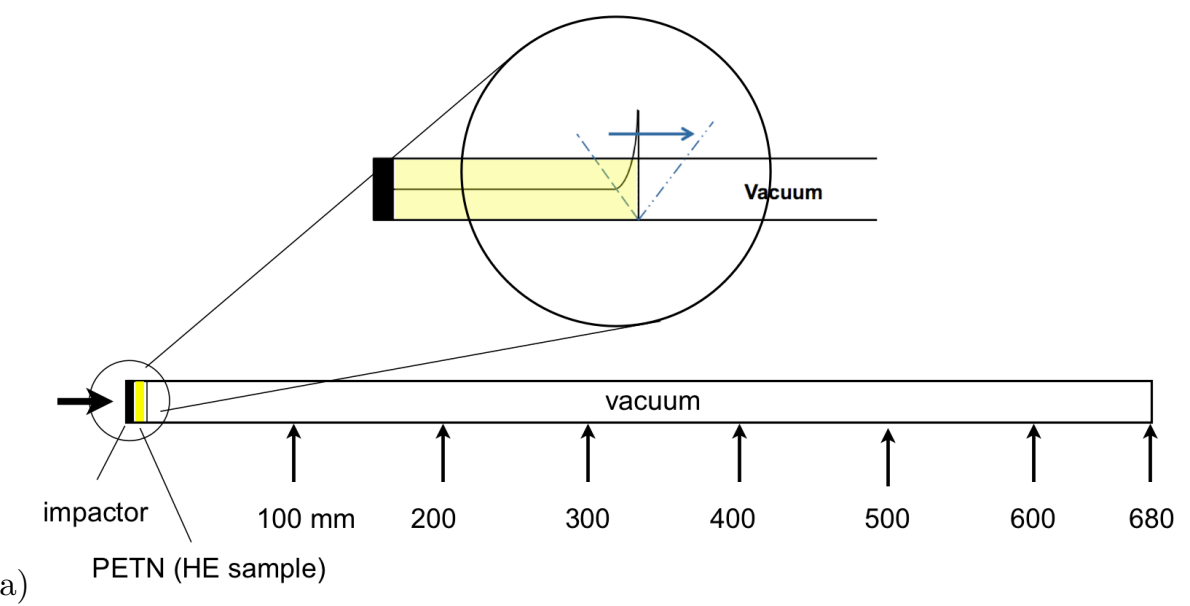

Figure 8: The location of impactor, PETN sample, vacuum and seven probe locations of measurement for simulation in Example

Figures 9 and 10 plot the state variables in the region $0<x<10 \mathrm{~mm}$. They show the formation of a detonation, followed by the initial expansion of products as they start to vent into the vacuum interface. Figure 9 shows the solution profiles of the state variables for Case $\mathrm{I}$ at the indicated times from $t=0$ to $t=0.69 \mu \mathrm{sec}$. The solution profiles at $\mathrm{t}=0.39 \mu \mathrm{sec}$ corresponds to a nominally steady CJ detonation wave that is established in the PETN sample, just prior to the detonation shock hitting the vacuum interface $x_{v}$ that is initially located at $4 \mathrm{~mm}$. Once the detonation shock wave hits the vacuum interface, the products expand into the vacuum via the action of a rarefaction wave. Figure 10 shows the corresponding contours of the state variables plotted in the x-t (space and time) for Case I. Figures 9(d) and 10(d), clearly show the region of interest for the experimental study where quenching of the reaction occurs and that would be the region where reactions of the intermediate species would likely cease, since the pressure in the rarefaction fan region drops rapidly.

Next we discuss the observations in the full length of the vacuum region $x \leq 680 \mathrm{~mm}$. After the sample is detonated, the products expand toward the vacuum and spread evenly in the entire vacuum region of the experiment. Figure 11(a) shows the density profiles in vacuum expanding process from times $t=5$ $\mu$ sec to $t=270 \mu \mathrm{sec}$ for Case I. The density was monitored at various locations. Figure $11(\mathrm{~b})$ shows the monitored density at the end of the vacuum region at $x=680 \mathrm{~mm}$ for Cases I, II and III. The density and time of arrival of the density is different for all three cases, and is affected by different sample thicknesses and impact velocities associated with each case. Figures 12 shows the pressure and particle velocity plotted in the $x-t$ (space, time) plane for the entire vacuum regions, for Case I. The velocity of vacuum interface can be estimated to be about $19 \mathrm{~mm} / \mu$ sec.

For all three cases at each probe location, we recorded the time of arrival (TOA) that corresponds to a time when specified density value was first sensed. The densities ranged from a low monitoring value of 
(a)

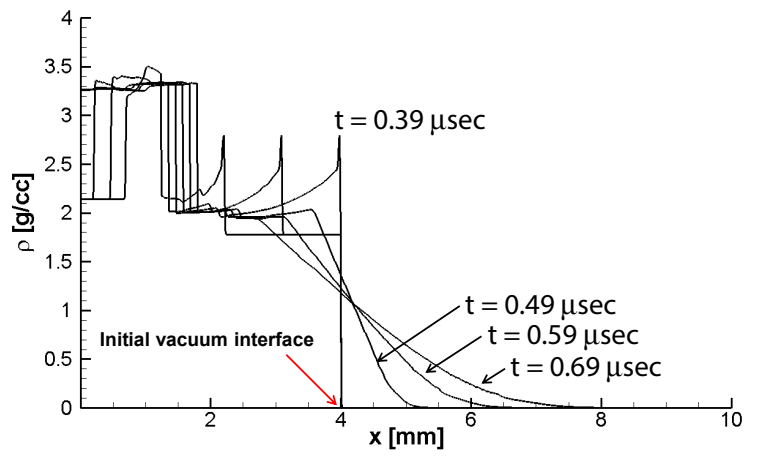

(b)

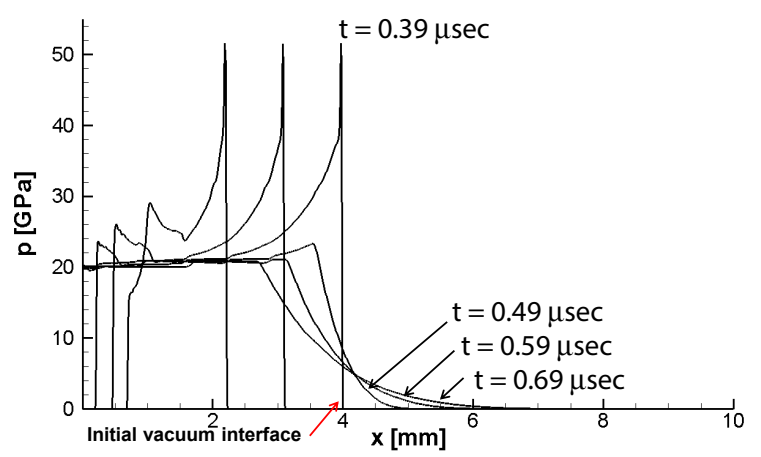

(c)

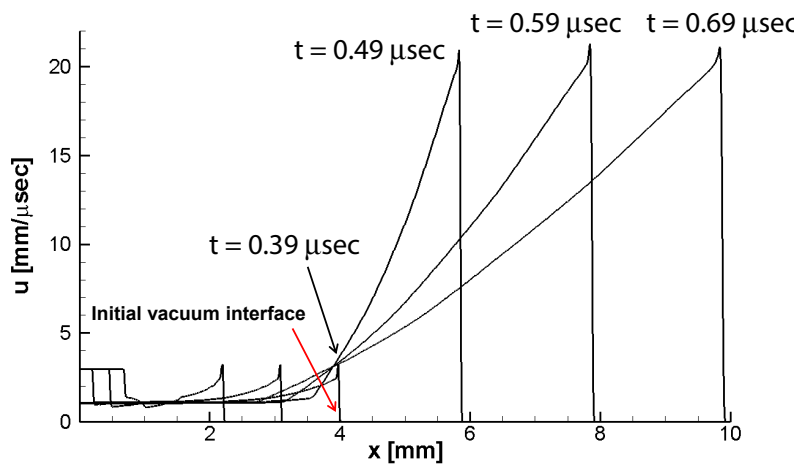

(d)

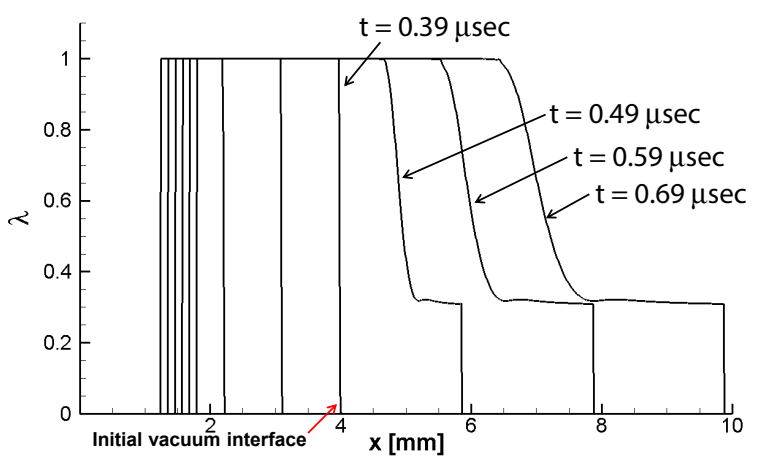

Figure 9: Time sequence s of detonation and expansion processes for Case I: (a) Density [g/cc], (b) Pressure [GPa], (c) Velocity $[\mathrm{mm} / \mu \mathrm{sec}],(\mathrm{d})$ Reaction Progress . 


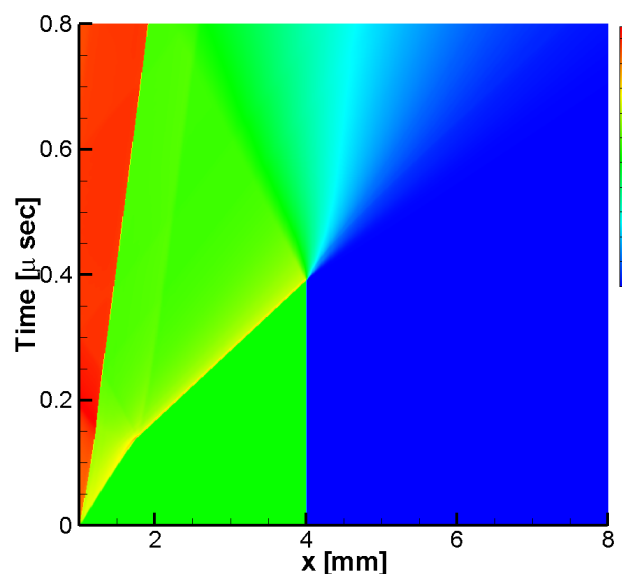

(a)

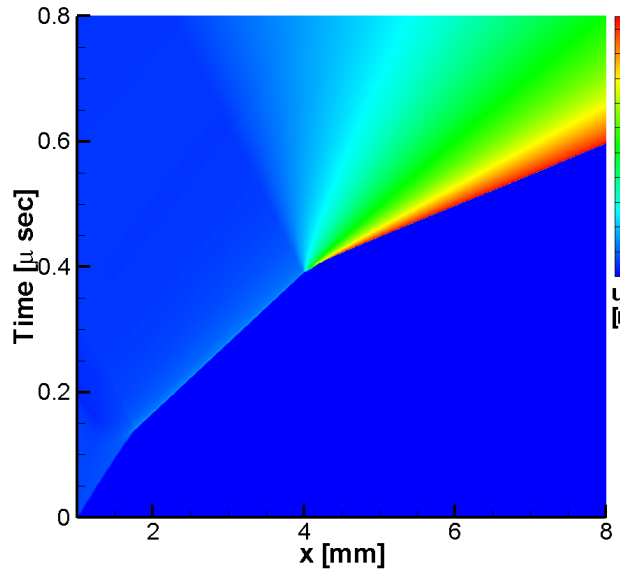

(c)

Figure 10: (a) Density, (b) Pressure $\left(\log _{10}(p)\right)$, (c) Velocity, (d) Reaction Progress field in space and time domain for Case I

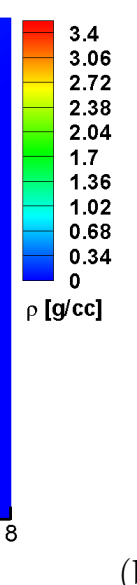

(b)
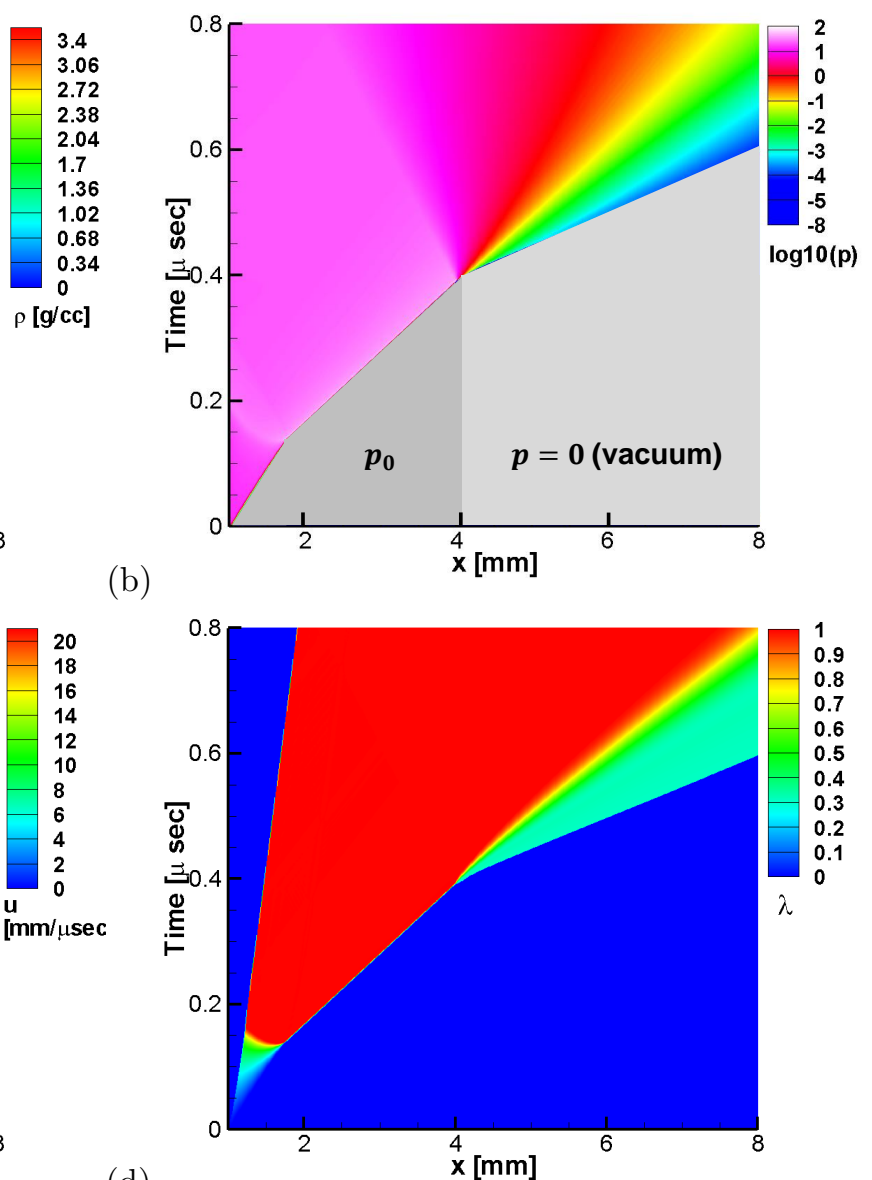

(d)

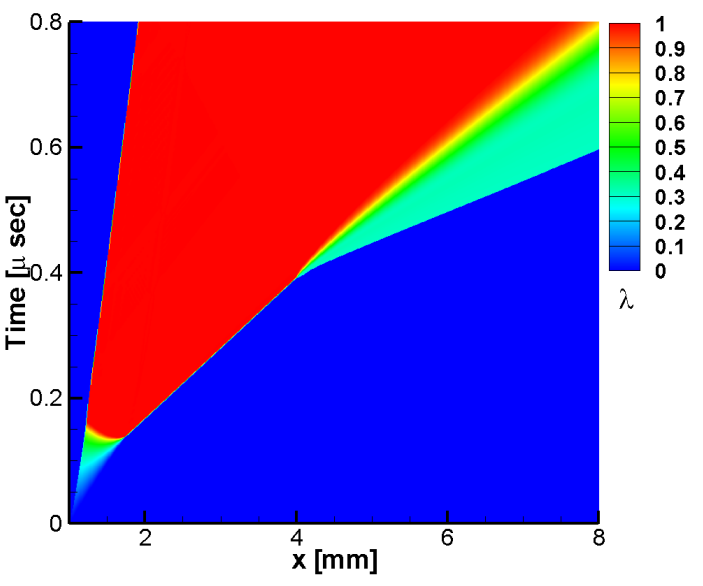

(a)

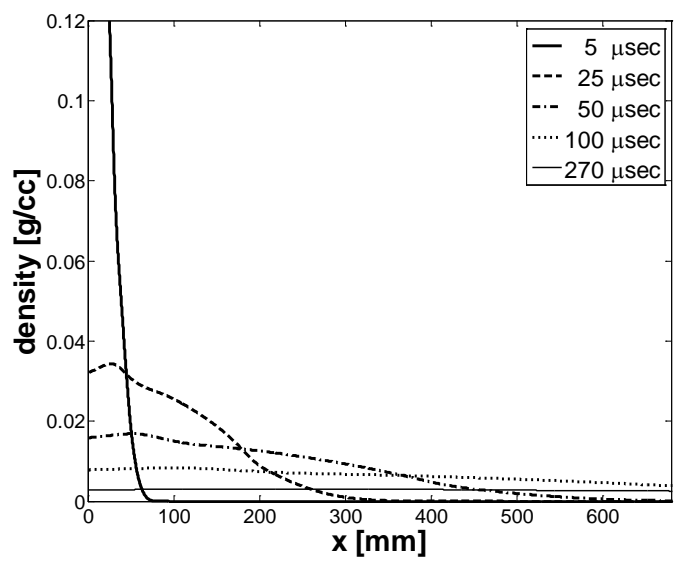

(b)

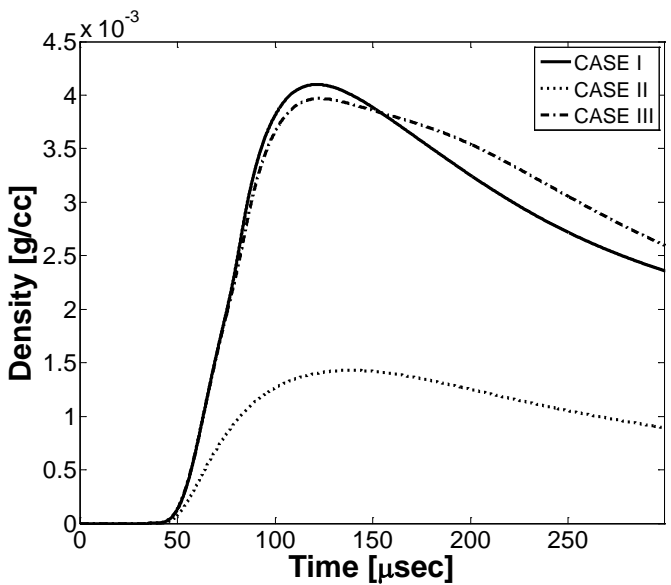

Figure 11: (a) Density profile expanding toward the vacuum region (b) Monitoring density at $x=680 \mathrm{~mm}$ for the Cases I-III .

$10^{-8} \mathrm{~g} / \mathrm{cc}$, to a maximum of $10^{-3} \mathrm{~g} / \mathrm{cm}^{3}$. The probes first senses the low values and later sense the higher 

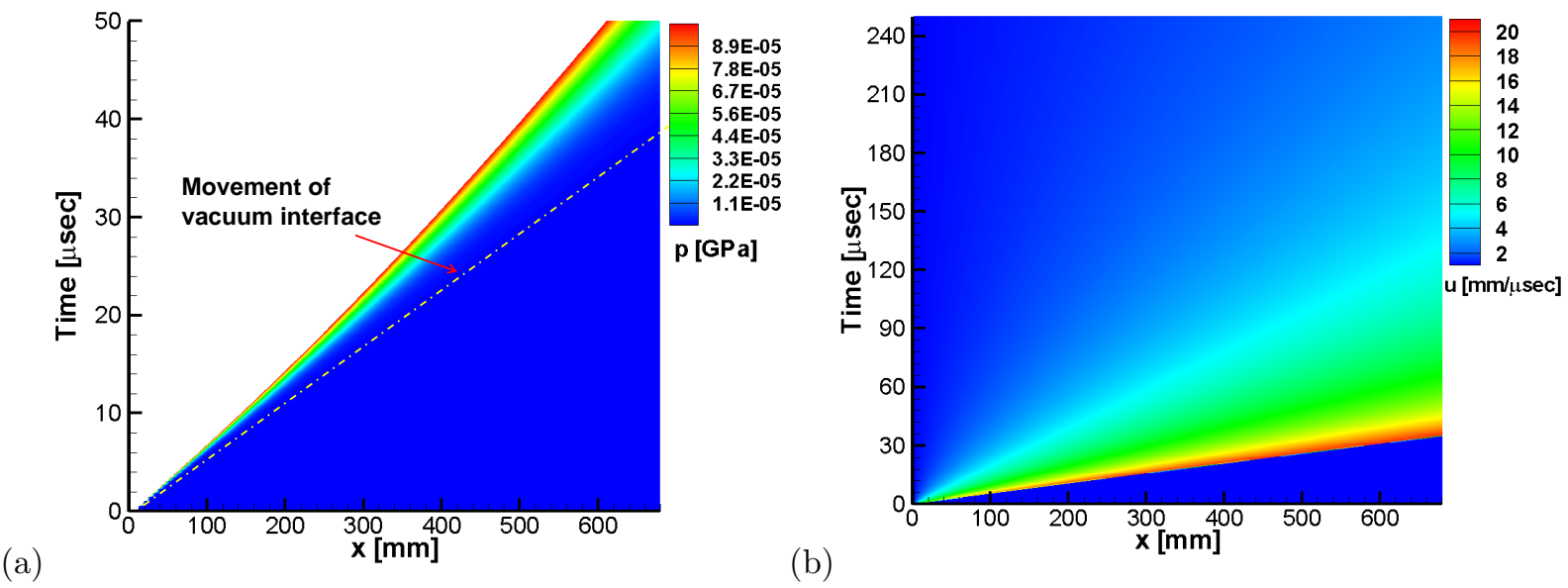

Figure 12: Pressure(a) and particle velocity(b) fields for Case I in $x-t$ domain .

values and the results for all three cases are shown in Figure 13. Case II has a smaller explosive sample thickness than Case I, but is subjected to the same impact velocity. In Case II, the detonation wave is not a steady state, CJ detonation when it hits the initial vacuum interface. Figure 11(b) shows that a smaller peak density is attained and that TOA at the monitoring locations has larger values. Thus the recorded velocity of a constant density front, measured by sampling the products expansion in the vacuum region is slower for Case II than for Case I. Case III has the same sample thickness of the PETN sample as Case I, but the sample is subject to a lower impact velocity. In Case III, the sample is thick enough so that a steady state CJ occurs, and the peak pressure and density observed at the probes is quite similar to Case I, but very slight shift of TOA of fixed density value at the probes is observed.

These results can be summarized by computing the phase velocity of a constant density front as the products encounter each probe. The results are listed in Table 4. The table shows that the velocity of the front, for a given monitored density, ranges from 8 to $19 \mathrm{~mm} / \mu \mathrm{sec}$ and is a function sample thickness and impact velocity. Experiments by Thomas et al. [32] showed that the detonation products expanding toward vacuum achieve terminal velocities in the range of 8 to $12 \mathrm{~mm} / \mu$ sec. Fajardo et al. [33], reported the leading-edge velocities of species through the vacuum approximately $\approx 10 \mathrm{~mm} / \mu \mathrm{sec}$ in their experiments on aluminum ablation. Lundborg [34] measured the velocity of the shock front of TNT evacuating from chambers and the velocity increased from $10 \mathrm{~mm} / \mu$ sec to $20 \mathrm{~mm} / \mu \mathrm{sec}$ with decreasing pressure. So our simulations easily fall in the range reported in related experiments, and is a reasonable check of the applicability of these algorithms and simulation techniques for the TOFMS experiments.

\begin{tabular}{|cc|c|c|c|}
\hline \multicolumn{2}{|c|}{ Monitoring density } & Case I & Case II & Case III \\
\hline $10^{-8}$ & $\mathrm{~g} / \mathrm{cc}$ & 19.64343 & 18.99816 & 19.67554 \\
\hline $10^{-7}$ & $\mathrm{~g} / \mathrm{cc}$ & 18.46885 & 17.87125 & 18.49775 \\
\hline $10^{-6}$ & $\mathrm{~g} / \mathrm{cc}$ & 17.31006 & 16.73676 & 17.34145 \\
\hline $10^{-5}$ & $\mathrm{~g} / \mathrm{cc}$ & 15.81749 & 15.27493 & 15.85946 \\
\hline $10^{-4}$ & $\mathrm{~g} / \mathrm{cc}$ & 13.76884 & 13.13290 & 13.82020 \\
\hline $10^{-3}$ & $\mathrm{~g} / \mathrm{cc}$ & 10.50874 & 8.14376 & 10.50174 \\
\hline
\end{tabular}

Table 4: Velocity $[\mathrm{mm} / \mu \mathrm{sec}]$ of monitored (specified) density front observed in the vacuum region for each Cases I, II and III .

\subsection{Comparison of two different closure models that are used to define the mixture EOS}

The vacuum tracking interface algorithms described in this paper can be generally used for simulating the propagation of products from the reaction of multi-component mixtures and can be modeled with any 


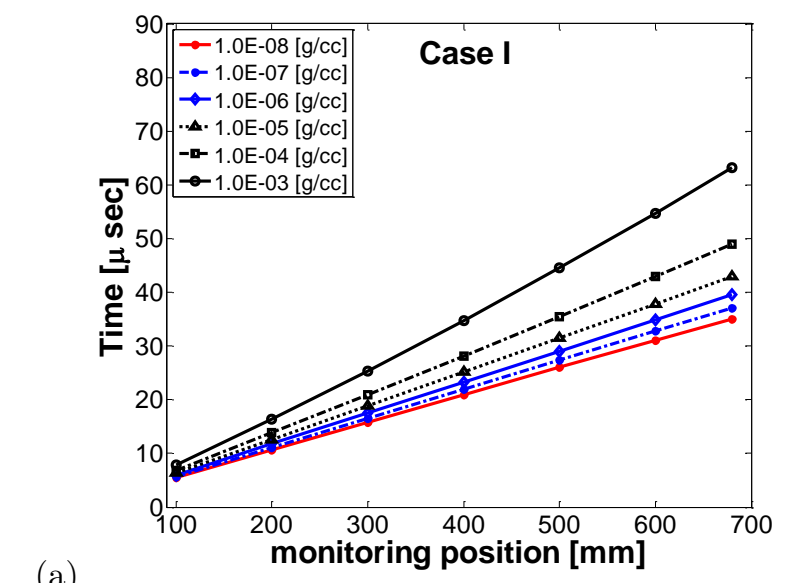

(a)

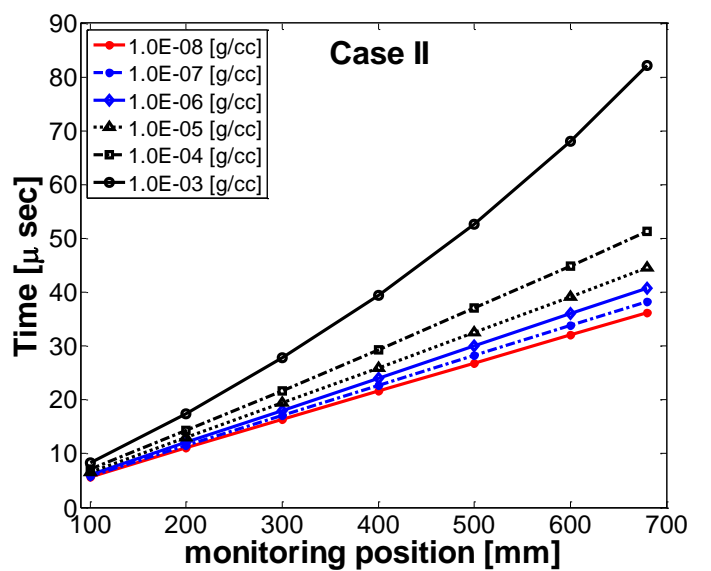

(b) monitoring position [mm]

(c)

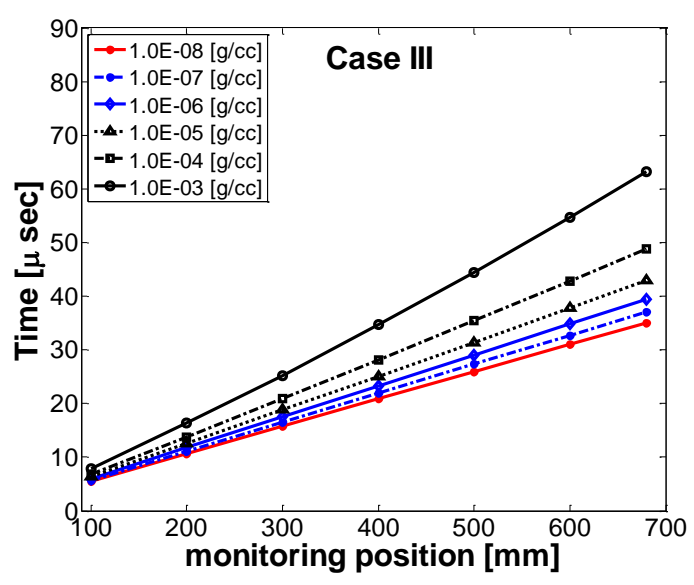

Figure 13: Time of arrival of quenched products at a specified density as a function of monitoring position for (a) Case I, (b) Case II, (c) Case III .

EOS that obeys the Liu-Smoller conditions for the mixture. However when a multi-component formulation is used for the mixture, one must provide some closure conditions that are in essence the statements of local equilibrium between components within the mixture. For a Gibbs formulation one posits a single pressure and temperature $(p, T)$ for the mixture, denoted as (PT-EQB). This means that the component EOS forms 
must also share the same mixture pressure and temperature. But one can close the mathematical formulation by proposing slightly different statements. A common one is that while there a common pressure (P-EQB), the ratio of the volumes of components is either constant or is a function of the composition, in which case the component volume are not pressure dependent.

This model (P-EQB) enforces only pressure equilibrium and specifies the volume component ratios as a closure condition. It is attractive since it uses EOS forms that are linear in the pressure and the EOS forms are closed explicitly. This type of closure eliminates the need for an iterative cycle to determine the temperature, and might be considered if one has two (or multi-) temperature model for reactants and products that are in pressure equilibrium but not temperature equilibrium. However our purpose here is simply to illustrate that changing the mixture closure condition does have an effect on the simulated results, since closure really represents a different model of the mixture EOS. We note that as we changed the closure models, the qualitative features of the simulated flow are quite similar but the computed values, that are important when interpreting chemical measurements made at the probes, do change.

To examine the dependencies of the simulation results on the mixture closure models, we implemented the pressure equilibrium (P-EQB) closure for the mixture equation of state in our PETN model and carried out the simulations for Case I and II. Comparisons of simulated results for Case I and II, that use the two closures (PT-EQB) and (P-EQB) are shown in Figure 14 and 15. The TOA in Figure 14 is plotted versus the probe locations. The detection velocities of the fixed density of $10^{-8} \mathrm{~g} / \mathrm{cc}$ for Case I are listed in Table 5. The velocities for the two closures, range respectively from 8 to $19 \mathrm{~mm} / \mu \mathrm{sec}$ and 9 to $13.5 \mathrm{~mm} / \mu \mathrm{sec}$.

Figure 15 shows a comparison of the density recorded at $x=680 \mathrm{~mm}$ for the two closure models. Both models show the same tendencies and approximately the same densities, however there are noticeable differences in the time shift and peak density at a fixed monitoring point. The pressure equilibrium (P-EQB) model shows higher peak density but slower time of arrival for Case I and II. These differences are entirely attributed to the differences in the closure models. Thus careful physical chemistry-based considerations must be taken into account to make accurate quantitative predictions for this class of simulations.

\begin{tabular}{|cl|c|c|}
\hline \multicolumn{2}{|c|}{ Monitoring density } & PT-Equilibrium & P-Equilibrium \\
\hline $10^{-8}$ & $\mathrm{~g} / \mathrm{cc}$ & 19.64343 & 13.47709 \\
\hline $10^{-7}$ & $\mathrm{~g} / \mathrm{cc}$ & 18.46885 & 12.90323 \\
\hline $10^{-6}$ & $\mathrm{~g} / \mathrm{cc}$ & 17.31006 & 12.28501 \\
\hline $10^{-5}$ & $\mathrm{~g} / \mathrm{cc}$ & 15.81749 & 11.54734 \\
\hline $10^{-4}$ & $\mathrm{~g} / \mathrm{cc}$ & 13.76884 & 10.57082 \\
\hline $10^{-3}$ & $\mathrm{~g} / \mathrm{cc}$ & 10.50874 & 8.952551 \\
\hline
\end{tabular}

Table 5: Comparison of the velocity determined by a monitoring density for closures, PT-EQB and PEQB [mm/ $\mu$ sec] for Case I

\section{Conclusions}

We have illustrated how the original Munz's vacuum tracking algorithm for a single ideal gas can be extended to a general multi-component reacting flow, with an Euler solver method that uses a linearized Riemann solver for a Mie-Gruneisen EOS form. Our implementation uses a fairly standard, cell based TVD scheme with source splitting to solve the reacting Euler equations. We have shown that for a non-ideal EOS model, our method can be implemented with different mixture closure models for pressure and temperature equilibrium, or just pressure equilibrium that uses an auxiliary condition such as a specified volume ratio to define the mixture equation of state. One must ensure that the Liu-Smoller conditions hold for the mixture EOS in the limiting case of a vacuum. The methods illustrated here are fairly robust and are easy to implement in any cell-based finite difference code framework. We feel that this is a welcome addition to a set of basic methods in an existing simulation code framework for interactions between multiple materials that are subjected to extreme pressure gradients. In cases where there is a violent expansion of material into 

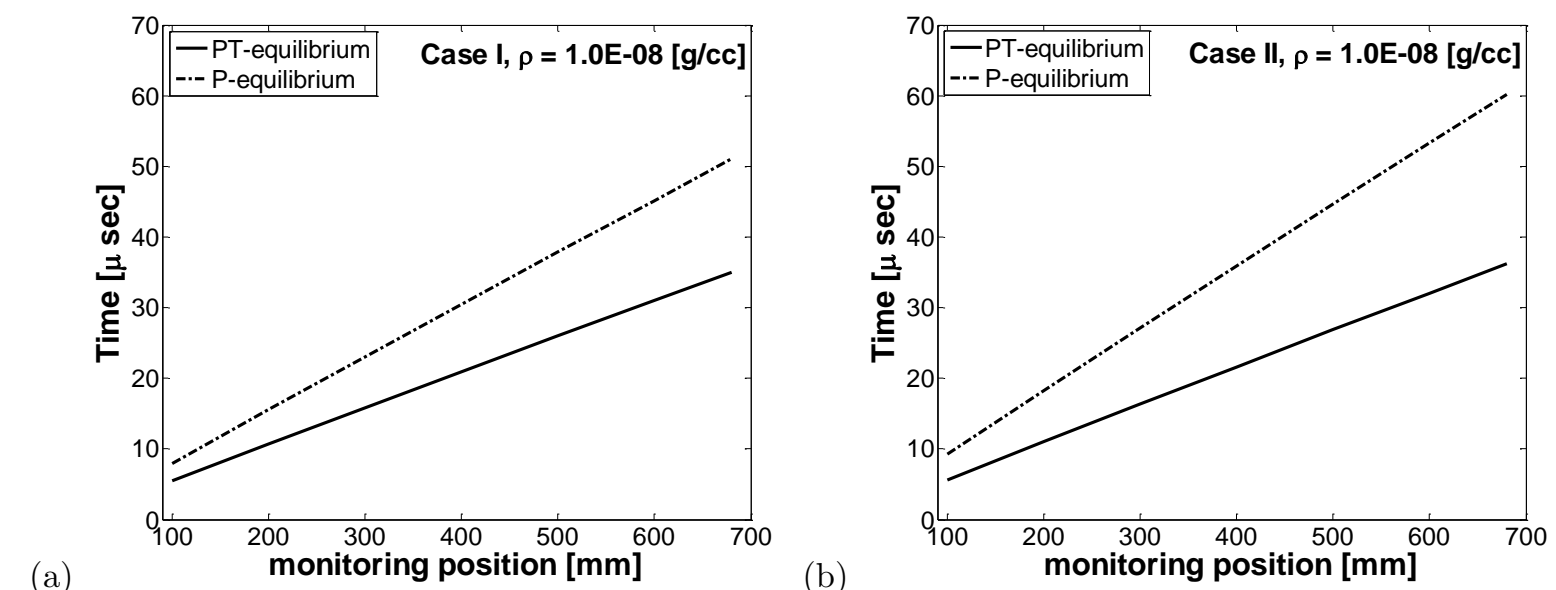

Figure 14: Comparison of the two different closures at probe locations: Monitoring TOA of front of density $10^{-8} \mathrm{~g} / \mathrm{cc}$ of (a) Case I and (b) Case II.

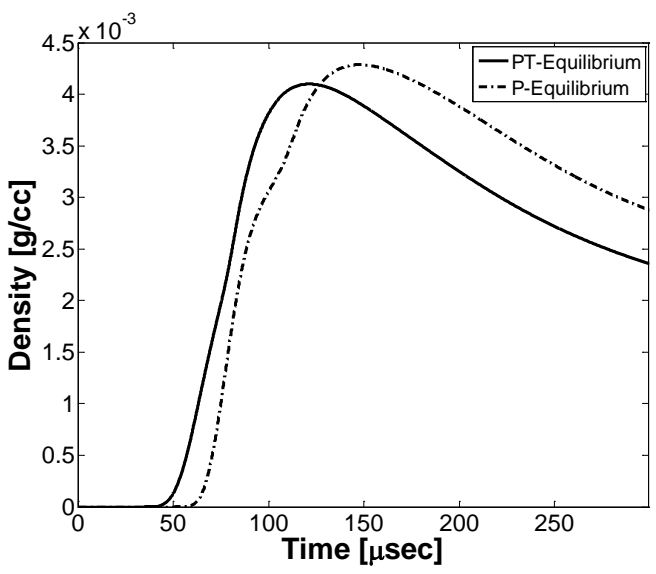

(a)

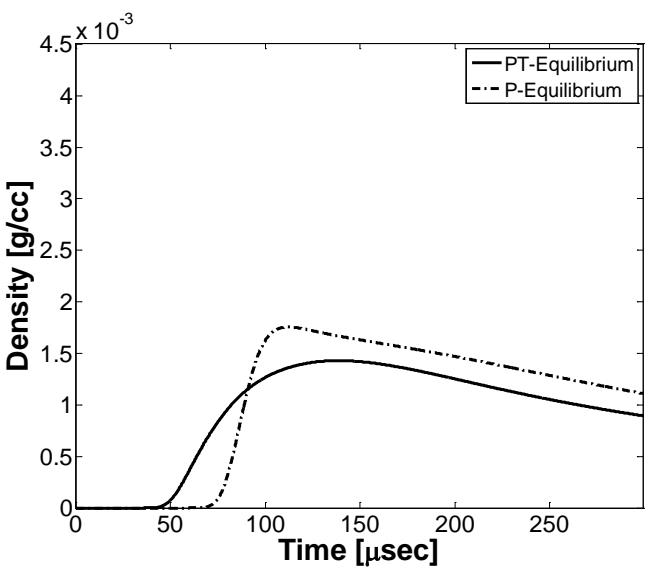

(b)

Figure 15: Comparison of the two different closures: The density at at $x=680 \mathrm{~mm}$ versus time for (a) Case I and (b) Case II.

vacuum or near vacuum regions it is essential to compute sensible and physically defined fluxes, as defined by the underlying models in order to avoid severe computational difficulties. Thus we believe the methodology presented here will have widespread applications and extensions to a much larger class of problems.

\section{Acknowledgments}

Supported by the US Air Force Research Laboratory, Munitions Directorate F08630-00-1-0002, and the Air Force Office of Scientific Research, Mathematics FA9550-06-1-0044. D. S. Stewart was also supported by the Defense Threat Reduction Agency, HDTRA1-10-1-0020.

\section{References}

[1] C.D. Munz. A tracking method for gas flow into vacuum based on the vacuum Riemann problem. Mathematical methods in the applied sciences, 17(8):597-612, (1994).

[2] E. Fossum, C. Molek, W. Lewis, and M. Fajardo. Benchtop energetics: Detection of hyperthermal species. Bulletin of the American Physical Society, 56, (2011)

[3] N. C. Blais, H. A. Fry, N. R. Greiner Apparatus for the mass spectrometric analysis of detonation products quenched by adiabatic free expansion. Review of Scientific Instruments, 64(1):174-183, (1993). 
[4] B. J. Lee, E. F. Toro, C.E. Castro, N. Nikiforakis. Adaptive Osher-type scheme for the Euler equations with highly nonlinear equations of state. Journal of Computational Physics, 264:165-183, (2013).

[5] P. Glaister. An approximate linearised Riemann solver for the Euler equations for real gases. Journal of Computational Physics, 74(2):382-408, (1988).

[6] S. Xu. Modeling and numerical simulation of deflagration-to-detonation transition in porous energetic materials. PhD. Dissertation, Theoretical and Applied Mechanics, University of Illinois, (1996).

[7] S. Xu, T. Aslam, D. S. Stewart. High resolution numerical simulation of ideal and non-ideal compressible reacting flows with embedded internal boundaries. Combustion Theory and Modelling, 1:113-142, (1997).

[8] E.F. Toro. Riemann solvers and numerical methods for fluid dynamics: A practical introduction. Springer Verlag, (2009).

[9] P. Colella, A. Majda, and V. Roytburd. Theoretical and numerical structure for reacting shock waves. Shock Waves, 7(4), (1986).

[10] T. Liu, J. Smoller. On the vacuum state for the isentropic gas dynamics equations. Advances in Applied Mathematics, $1: 345-359,(1980)$.

[11] H. B. Callen. Thermodynamics and an introduction to thermostatistics John Wiley \& Sons. New York, (1985).

[12] W. Fickett and W.C. Davis. Detonation: Theory and experiment. Dover Pubns, (2011).

[13] L. E. Fried, W. M. Howard, P. C. Souers, and P. A. Vitello. Cheetah 6.0 User Manual. Lawrence Livermore National Laboratory, Livermore, CA, LLNL-SM-416166, (2010).

[14] E. L. Lee, C. M. Tarver. Phenomenological model of shock initiation in heterogeneous explosives. Physics of Fluids (1958-1988), 23(12):2362-2372, (2008).

[15] A. C. Hindmarsh. Lsode and lsodi, two new initial value ordinary differnetial equation solvers. ACM Signum Newsletter, $15(4): 10-11,(1980)$.

[16] A. Harten, B. Engquist, S. Osher, S.R. Chakravarthy. Uniformly high order accurate essentially non-oscillatory schemes, iii. Journal of Computational Physics, 71(2):231-303, 1987.

[17] H. Deconinck, T..J. Barth. High-order methods for computational physics, volume 9. Springer Verlag, (1999).

[18] X.D. Liu, S. Osher, T. Chan. Weighted essentially non-oscillatory schemes. Journal of Computational Physics, 115(1):200212, (1994).

[19] P. L. Roe. The use of the Riemann problem in finite difference schemes. In Seventh International Conference on Numerical Methods in Fluid Dynamics, pages 354-359. Springer, (1981).

[20] P. L. Roe. Approximate Riemann solvers, parameter vectors, and difference schemes. Journal of Computational Physics, 43(2):357 - 372, (1981).

[21] E. Halter and E. Martensen. A fast solver for Riemann problems. Mathematical methods in the applied sciences, 7(1):101$107,1985$.

[22] R. Menikoff. Complete Mie-Gruneisen equation of state. Los Alamos Technical Report LA-UR-12-22592, Los Alamos National Laboratory, May (2012).

[23] D.S. Stewart, S. Yoo, and W.C. Davis. Equation of state for modeling the detonation reaction zone. In 12th Symp.(Intl) on Detonation, pages 1-11, (2002).

[24] W. Romberg. Vereinfachte numerische Integration. Det Kongelige Norske Videnskabers Selskab Forhandlinger, 28(7):30$36,(1955)$.

[25] W. L. Ng, J. E. Field, H. M. Hauser. Thermal, fracture, and laser-induced decomposition of pentaerythritol tetranitrate. Journal of Applied Physics, 59(12):3945-3952, (1986).

[26] C. M. Tarver, R. D. Breithaupt, J. W. Kury. Detonation waves in pentaerythritol tetranitrate. Journal of Applied Physics, 81(11):7193-7202, (1997).

[27] D. Stirpe, J. O. Johnson, and J. Wackerle. Shock initiation of XTX-8003 and pressed PETN. Journal of Applied Physics, 41(9):3884 -3893, Aug. (1970).

[28] V.I. Korepanov, V.M. Lisitsyn, V.I. Oleshko, V.P. Tsypilev. Petn detonation initiated by a high-power electron beam. Technical Physics Letters, 29:669-671, (2003).

[29] J. B. Bdzil and D. S. Stewart. The dynamics of detonation in explosive systems*. Annu. Rev. Fluid Mech., 39:263-292, (2007).

[30] J. B. Bdzil, D. S. Stewart Theory of detonation shock dynamics. Shock Waves Science and Technology Library, Vol. 6:pp 373-453, (2012).

[31] D. S. Stewart, S. Yoo, B. L. Wescott. High-order numerical simulation and modelling of the interaction of energetic and inert materials. Combustion Theory and Modelling, 11(2):305-332, (2007).

[32] R. L. Kovach T. J. Ahrens, C. F. Allen. Explosive gas blast: The expansion of detonation products in vacuum. Journal of Applied Physics, 42(2):815-829, (1971).

[33] M. Fajardo, E. C. Fossum, C. D. Molek, and W. K. Lewis. Benchtop energetics progress. AIP Conference Proceedings, 1426(1):217-222, (2012).

[34] N. Lundborg. Front and mass velocity at detonation in evacuated chambers. Arkiv For Fysik, 25(6):541, (1964). 